\title{
Spiral inertial waves emitted from geophysical vortices
}

\author{
Peng Wang*1 and Tamay M. Özgökmen \\ Rosenstiel School of Marine and Atmospheric Science, \\ University of Miami, Miami, FL 33149 \\ submitted to Ocean Modelling
}

${ }^{1 *}$ Corresponding author: pwang@rsmas.miami.edu

(C) 2016. This manuscript version is made available under the Elsevier user license http://www.elsevier.com/open-access/userlicense/1.0/ 
By numerically simulating an initially unstable geophysical vortex, we discover for the first time a special kind of inertial waves, which are emitted in a spiral manner from the vortices; we refer to these waves as spiral inertial waves (SIWs). SIWs appear at small Rossby numbers $(0.01 \leq R o \leq 1)$ according to our parameter sweep experiments; the amplitude, wavelength and frequency of SIWs are sensitive to Rossby numbers. We extend the Lighthill-Ford radiation into inertial waves, and propose an indicator for the emission of inertial waves; this indicator may be adopted into general circulation models to parameterize inertial waves. Additionally, in our tracer releasing experiments, SIWs organize tracers into spirals, and modify the tracer's local rate of change by advecting tracers vertically. Further, the spirals of SIWs resembles some spiral features observed in the ocean and atmosphere, such as spiral ocean eddies and spiral hurricane rainbands; thus, SIWs may offer another mechanism to form spiral eddies and rainbands. Since no density anomaly is required to generate the spirals of SIWs, we infer that the density anomaly, hence the baroclinic or frontal instability, is unlikely to be the key factor in the formation of these spiral features. Key words: Lighthill-Ford Radiation, Vortex Rossby Wave, Spiral Eddy, Spiral Rainband 


\section{Introduction}

Inertial waves can arise in rotating homogeneous fluids due to the Coriolis force (e.g. Greenspan 1969), and have been observed in different geometries, including the cylinder, sphere and cube (e.g. Fultz 1959; McEwan 1970; Zhang et al. 2001; Kelly et al. 2007; Messio et al. 2008; Lopez and Marques 2011; Boisson et al. 2012). Inertial waves are involved in geophysics, astrophysics and engineering, such as oceanic and atmospheric flows, liquid planet cores, and spacecraft fuel tanks (e.g. Aldridge and Lumb 1987; Manasseh 1992; Tilgner 2007; Noir et al. 2009; Cortet et al. 2010; Bordes et al. 2012).

The dispersion relation of inertial waves is $\omega^{2}=f^{2} m^{2} / K^{2}$ expressed in Cartesian coordinates, where $\omega$ is the frequency, $f$ is the Coriolis parameter, $m$ is the vertical wavenumber and $K$ is the total wavenumber; meanwhile, the dispersion relation of inertia-gravity waves is $\omega^{2}=\left(f^{2} m^{2}+N^{2} K_{h}^{2}\right) / K^{2}$, where $N$ is the buoyancy frequency and $K_{h}$ is the horizontal wavenumber. Clearly, the inertial wave is an extreme of inertia-gravity wave in the limit of $(N=0)$ zero buoyancy frequency (Gill 1982); consequently, we expect that the generation mechanisms of inertia-gravity waves may also fit inertial waves. Such mechanisms include the geostrophic/Rossby adjustment (e.g. Rossby 1938), Lighthill-Ford radiation (Lighthill 1952; Ford 1994a, 1994b; Ford et al. 2000, 2002; McIntyre 2009), spontaneous balance adjustment (e.g. O’Sullivan and Dunkerton 1995; Zhang et al. 2000, 2001; Zhang 2004; Wang and Zhang 2010), unbalanced instabilities (e.g. Molemaker et al. 2005; Plougonven et al. 2005; Gula et al. 2009; Vanneste 2013). In addition, the Lighthill-Ford radiation was extended to a forced-dissipative unsteady jet (Sugimoto et al. 2007; Sugimoto et al. 2008) and a spherical domain (Sugimoto and Ishii 2012).

Material dispersions (e.g. pollution dispersion, nutrient exchange) are of particular interest in geophysical flows, and has been extensively studied by means of Lagrangian particles, passive tracers and dynamical system approaches (e.g. Ledwell et al. 2008; Özgökmen et al. 
2011; Özgökmen et al. 2012; Ohlmann et al. 2012; Chabreyrie and Llewellyn Smith 2014; Rypina et al. 2014). In this study, we investigate whether material dispersions are affected by inertial waves.

By simulating an initially single geophysical vortex in a cylindrical domain, we discover a special kind of inertial waves emitted from the vortices. This kind of inertial waves has horizontal spirals; we refer to them as spiral inertial waves (SIWs). In fact, spiral waves have been reported in studies of inertia-gravity wave radiation (e.g. Schecter and Montgomery 2006; Viúdez 2006, 2007; Schecter 2008; Sugimoto et al. 2015), but for inertial waves, we believe it is the first time that the spirals have been discovered. It is worth mentioning that gravity waves are removed from our numerical simulations, because of the conditions of homogeneous density, rigid lid and flat bottom.

The paper is organized as follows. The numerical model is described in section 2. Examples of SIWs emission are presented in section 3.1. Parameter-sweep experiments reported in section 3.2 show the dependence of SIWs on Rossby numbers. In section 3.3, we extend the Lighthill-Ford radiation, and propose an indicator for the emission of inertial waves. The effects of SIWs on material dispersion are investigated in section 3.4. Finally, we summarize and discuss the main results in section 4 .

\section{Numerical model}

SIWs are simulated with NEK5000, which is a numerical solver for Navier-Stokes equations based on the spectral element method (e.g. Patera 1984; Fischer 1997). NEK5000 has been used in studying geophysical flows, including ocean mixed-layer instabilities (e.g. Özgökmen et al. 2011; Özgökmen et al. 2012), chaotic advection inside an ocean eddy (Pratt et al. 2014; Rypina et al. 2015) and effects of instabilities on 3D material transport within an ocean eddy (Wang and Özgökmen 2015). 
NEK5000 solves the following dimensionless equations of motion:

$$
\begin{aligned}
& \frac{\partial \mathbf{u}}{\partial t}+(\mathbf{u} \cdot \nabla) \mathbf{u}=-\nabla p-\frac{1}{R o} \hat{\mathbf{k}} \times \mathbf{u}+\frac{1}{R e} \nabla^{2} \mathbf{u}, \\
& \nabla \cdot \mathbf{u}=0 .
\end{aligned}
$$

The Rossby number is defined as $R o=U /(f L)$, where $U$ and $L$ are the characteristic scales of horizontal velocity and horizontal length; $f=2 \Omega$ is the Coriolis parameter, with $\Omega$ being the angular velocity of the background rotation. In the following experiments of vortex, we choose the maximum azimuthal velocity and the vortex radius as the $U$ and $L$, respectively; for all experiments, the $U$ and $L$ are similar, and therefore $R o$ is mainly determined by $f$. The Reynolds number is given by $R e=U L / \nu$, where $\nu$ is the kinematic viscosity. $\mathbf{u}=(u, v, w)$ is the relative velocity measured in the rotating frame, and $p$ is the pressure. $\hat{\mathbf{k}}$ is the unit vector in the vertical direction, and operator $\nabla=\left(\frac{\partial}{\partial x}, \frac{\partial}{\partial y}, \frac{\partial}{\partial z}\right)$. In addition, NEK5000 integrates the advection-diffusion equation (2) for the passive scalar (tracer):

$$
\frac{\partial C}{\partial t}+(\mathbf{u} \cdot \nabla) C=\frac{1}{P e} \nabla^{2} C
$$

where $P e=U L / \kappa$ is the Péclet number and $\kappa$ is the diffusivity of passive scalar $C$.

The numerical domain (Fig. 1 ) is a cylinder with radius $R=4$ and height $H=1$. The cylinder is filled with homogeneous fluid, which is in solid-body rotation with an angular velocity of $\Omega$ about its central axis. Instantly at time $=0$, a barotropic vortex is initialized in the center of the domain. The initial vortex has two types of vortical flows (Fig. 2) initialized by Eq. (3); Wang and Özgökmen (2015) investigated the stability of the two types of flows at different parameter $(R o, R e)$ regimes. At proper parameters $(R o, R e)$, the vortex defined by Eq. (3a) favors instabilities, producing a family of non-axisymmetric vortices; yet, the 
Table 1:

List of the numerical experiments. The vortices initialized by Eq. (3a) and Eq. (3b) are designated as target $(\mathrm{T})$ and control $(\mathrm{C})$ experiments, respectively. The initial vortex in Exp. T10 is anticyclonic, denoted by -Eq. (3a).

\begin{tabular}{lrcllc}
\hline Exp. \# & Initial vortex & Domain radius & $\boldsymbol{R o}$ & $\boldsymbol{R e}$ & Resolution \\
\hline \hline $\mathrm{C} 1$ & Eq. (3b) & 4 & 0.2 & $10^{4}$ & 10 \\
\hline $\mathrm{T} 1$ & Eq. (3a) & 4 & 0.2 & $10^{4}$ & 10 \\
\hline $\mathrm{T} 2$ & Eq. (3a) & 4 & 0.01 & $10^{4}$ & 10 \\
\hline $\mathrm{T} 3$ & Eq. (3a) & 4 & 0.04 & $10^{4}$ & 10 \\
\hline $\mathrm{T} 4$ & Eq. (3a) & 4 & 0.1 & $10^{4}$ & 10 \\
\hline $\mathrm{T} 5$ & Eq. (3a) & 4 & 0.4 & $10^{4}$ & 10 \\
\hline $\mathrm{T} 6$ & Eq. (3a) & 4 & 0.6 & $10^{4}$ & 10 \\
\hline $\mathrm{T} 7$ & Eq. (3a) & 4 & 1 & $10^{4}$ & 10 \\
\hline $\mathrm{T} 8$ & Eq. (3a) & 4 & 5 & $10^{4}$ & 10 \\
\hline $\mathrm{T} 9$ & Eq. (3a) & 8 & 0.2 & $10^{4}$ & 10 \\
\hline $\mathrm{T} 10$ & - Eq. (3a) & 4 & 0.2 & $10^{4}$ & 10 \\
\hline $\mathrm{T} 11$ & Eq. (3a) & 4 & 0.2 & $10^{4}$ & 13 \\
\hline $\mathrm{T} 12$ & Eq. (3a) & 4 & 0.2 & $2 \times 10^{4}$ & 13 \\
\hline
\end{tabular}

The boundary conditions are free-slip on the sidewall and no-slip at the bottom; on the top boundary, the velocity is prescribed with the same profile of the initial vortex. Note that the conditions of homogeneous density, rigid lid and flat bottom will exclude gravity waves.

The domain is discretized into $K=2560$ quadrilateral elements; on each element, solutions are approximated by Legendre polynomials of order $N=10$, which generates $K(N+1)^{3}=$ 3,407,360 grid points; for fixed $K$, increasing $N$ will increase the resolution. The numerical model is integrated for a total dimensionless time of 300 (about five eddy-turnover times) with a time step of $10^{-3}$. For the experiments listed in Table 1, the flows typically need an integration time of 120 150 to reach a steady state of total kinetic energy (Fig. 3).

$$
\begin{aligned}
& V(r)=2 r \exp \left(-12 r^{8}\right), \\
& V(r)=3.3 r \exp \left(-2 r^{2}\right) .
\end{aligned}
$$

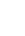


By comparing the two contrasting experiments $\mathrm{T} 1$ and $\mathrm{C} 1$, we illustrate how the stability and axisymmetry of the vortex can affect the emission of SIWs. The parameter-sweep experiments $\mathrm{T} 2-\mathrm{T} 8$ are designed to investigate the dependence of SIWs on Rossby numbers; the range of $R o$ is from 0.01 to 5 , which covers, for example, ocean mesoscale to submesoscale eddies. We use Exp. T9 to explore the effect of domain size on SIWs, and employ Exp. T10 to study the SIWs emitted from an initially anticyclonic vortex. In order to check the numerical convergence, we conduct Exps. T11 and T12 on a higher-resolution mesh (by increasing the polynomial order to $N=13$, yielding $7,024,640$ grid points) and with a larger Reynolds number $\left(R e=2 \times 10^{4}\right)$.

\section{Results}

\subsection{Emission of SIWs}

Take Exp. T1 as an example to illustrate the generation of SIWs by the initially cyclonic vortex. The vertical vorticity (Figs. 4a,c,e) demonstrates the vortex evolution; the vertical velocity (Figs. 4b,d,f) manifests the emitted inertial waves. Besides, the inertial waves can also be manifested by the vorticity anomaly, horizontal divergence and vertical gradient of pressure.

In reality, many factors can push vortices away from geostrophic balance; these include wind forcing, interaction with other vortices, internal waves, bathymetry, etc. In this sense, it is useful to investigate how an initially unbalanced vortex evolves. In our experiments, after the unbalanced vortex is initialized, the geostrophic adjustment takes place, leading to the emission of circular inertial waves (Fig. 4b). Later, four satellite vortices with negative vorticity are created by the azimuthal wavenumber- 4 mode of barotropic instability (Fig. 4c); they surround the vortex core of positive vorticity. This instability is caused by the interactions of phase-locking vortex Rossby waves, which exist due to the gradient of mean 
flow's potential vorticity (e.g. Montgomery and Kallenbach 1997; Schecter and Montgomery 2004). Instabilities (or vortex Rossby waves) change the initially axisymmetric vortex into non-axisymmetric, breaking the circular pattern of inertial waves. Subsequently, four pairs of SIWs are emitted from these non-axisymmetric vortices; each pair consists of two spirals with opposite signs in vertical velocity (Fig. 4d). Then the four satellite vortices merge and form two larger ones. At the final stage of vortex evolution, a familiy of vortices constituted by a central vortex and two satellite vortices, also known as tripolar vortices, is established (Fig. 4e); meanwhile, SIWs have organized the spirals into two groups (Fig. 4f). In addition, the total kinetic energy has now reached a steady state, and the flow becomes nearly balanced, rather than exactly balanced, for it still continuously emits SIWs. Consequently, we attribute the wave emission at the final stage to the spontaneous adjustment emission that is responsible for the wave emission from balanced flows; in contrast, the geostrophic adjustment accounts for the wave emission from unbalanced flows (e.g. Plougonven and Zhang 2014). Typically, the geostrophic adjustment makes flows towards a balanced state free of wave emission, while the spontaneous emission drives flows to emit waves at any stage of evolution (e.g. Sugimoto et al. 2008).

Moreover, the emitted waves are so weak relative to the vortices that no significant back-reaction between waves and vortices are expected. In fact, we only observe a weak back-reaction during the vortex merging, which merely lasts a short period; therefore, the back-reaction is negligible in our experiments.

Our experiments show that SIWs have two organized phases. The first phase (Fig. 4d) is associated with the wavenumber-4 barotropic instability; accordingly, this phase consists of four complete waves in the azimuthal direction (Fig. 5a). The second phase (Fig. 4f) contains two wave packets (Fig. 5b), which are associated with the two satellite vortices formed by the vortices merging. We refer to the first and second phase as "SIW-4" and "SIW-2", respectively. The SIW-4 phase is transient and is soon replaced by the SIW-2, 
which keeps intact till the end.

Also, the emission of inertial waves is illustrated by the Hovmöller diagram (Fig. 6), which reveals the radially-outward propagation of wave energy (group velocity) due to the outgoing wave emission. As waves approach the far field, wave energy is gradually dissipated. The zoom-in views of Hovmöller diagram (Figs. 6b,c) reveal the radially-inward propagation of phase speed; this inward phase propagation is consistent with the inward spirals of SIWs ( $c f$. Figs. $4 \mathrm{~d}, \mathrm{f}$ ), if facing the direction of the cyclonically rotating vortices.

The distance that inertial waves can propagate away from the vortices is limited by the domain size; therefore, we design Exp. T9 which is performed in a domain with doubled radius, to inspect the effect of domain size on inertial waves. As shown in Fig. 7, SIWs still exhibit spiral patterns similar to that observed in Exp. T1 (cf. Figs. 4d,f), and also have two organized phases of SIW-4 and SIW-2. However, the spirals of SIWs extend longer in the larger domain; consequently, we infer that if the domain radius expands more, the spirals will extend even longer.

An initially anticyclonic vortex in Exp. T10 also emits SIWs (Fig. 8), and develops similar processes as observed in the cyclonic vortex, such as the geostrophic adjustment, vortex Rossby waves, vortex merging and spontaneous wave emission. As well, these SIWs exhibit a transient phase of SIW-4 (Fig. 8d) and a final phase of SIW-2 (Fig. 8f); nevertheless, the direction of spirals has reversed.

By contrast, the vortex in Exp. C1 remains axisymmetric and stable throughout the experiment, but no SIWs are observed (Fig. 9). At the early stage of evolution, the geostrophic adjustment also generates circular inertial waves; however, these waves are gradually diminishing while the flow is approaching a steady state. The fact that no SIWs are generated by this stable, axisymmetric vortex indicates that the generation of SIWs 
requires the vortex to become non-axisymmetric and to remain unsteady (i.e. the local time derivative $\frac{\partial}{\partial t} \neq 0$ ). Particularly, the non-axisymmetry is necessary in forming the spirals, and the unsteadiness is requisite in generating waves.

The numerical convergence is confirmed by the similar wave characteristics observed in a higher-resolution mesh with a larger Reynolds number. For instance, in comparison with the SIWs in Exp. T1 (cf. Figs. 4d,f and Fig. 5), the SIWs in Exps. T11 and T12 (Fig. 10) have similar wave patterns, nearly equal amplitudes, and same organizations like phases SIW-4 and SIW-2. Particularly in Exp. T12, the wave amplitude is a little larger at phase SIW-4, and the wave is a bit more intense in the far field due to the larger Reynolds number (smaller viscosity). Together with the fact that the wave scale is much larger than the grid spacing, the numerical convergence rules out the possibility that SIWs are numerical artifacts.

\subsection{Dependence of SIWs on Rossby number}

In order to investigate how SIWs change with Rossby numbers, we resort to parameter-sweep experiments T2-T8. In experiments T2-T7 (small $R o \leq 1$ ), circular inertial waves are observed owing to the geostrophic adjustment at the early stage of evolution; later, the circular inertial waves are replaced by SIWs. At the final stage, all SIWs evolve into the phase SIW-2 (Fig. 11). However, in Exp. T8 (large Ro=5), no inertial waves appear, and both the vorticity and velocity fields looks chaotic, lacking the tripolar structure observed in Exps. T2-T7.

Next we compare the wave frequency measured in experiments with the prediction by theory. The theoretical eigenfrequency or dispersion relation of inertial waves that exist in cylindrical domains, was first derived by Kelvin (1880) in a linear inviscid system. A complete derivation was documented by Greenspan (1969). Concisely, by the asymptotic expansion of small parameter, inertial waves are formulated as ageostrophic modes of an initial-value problem for rotating fluids. Then by exploiting proper boundary conditions of 
the cylindrical geometry, the dispersion relation is derived as

$$
\lambda_{n m k}=2\left(1+\frac{\xi_{n m k}^{2}}{n^{2} \pi^{2} a^{2}}\right)^{-\frac{1}{2}}
$$

where $\lambda_{n m k}$ is the eigenfrequency of wave mode $(n, m, k)$ with $n, m, k$ denoting the axial, radial and azimuthal wavenumbers, respectively (Greenspan 1969). Here $a$ is the ratio of domain's radius to height, and $\xi_{n m k}$ is the $m$-th positive solution of the transcendental equation (5):

$$
\xi \frac{\mathrm{d}}{\mathrm{d} \xi} J_{|k|}(\xi)+k\left(1+\frac{\xi^{2}}{n^{2} \pi^{2} a^{2}}\right)^{\frac{1}{2}} J_{|k|}(\xi)=0
$$

where $J_{|k|}(\xi)$ is the Bessel function of the first kind of order $|k|$. The eigenfrequency $\lambda_{n m k}$ in Eq. (4) is normalized by the angular velocity $\Omega$ of background rotation, and has a maximum equal to 2 ; that is, the frequency of inertial waves cannot exceed the Coriolis parameter $f$ $(=2 \Omega)$.

We use spectral analysis to acquire the most energetic frequency and wavenumbers $(n, m, k)$ of waves emitted during the final stage of vortex evolution; they are referred to as experimental frequency and wavenumbers. Then we substitute $(n, m, k)$ into Eq. (5) to obtain $\xi$, which is used in Eq. (4) to calculate the theoretical frequency $\lambda$. As shown in Table 2, there is a good agreement between the theory and experiments. 
Table 2:

List of the most energetic mode and frequency of SIWs.

\begin{tabular}{llclc}
\hline \multirow{2}{*}{ Exp.\# } & \multirow{2}{*}{$\boldsymbol{R o}$} & \multirow{2}{*}{ Mode $(\boldsymbol{n}, \boldsymbol{m}, \boldsymbol{k})$} & \multicolumn{2}{c}{ Eigenfrequency } \\
\cline { 4 - 5 } & & & Theory & Experiment \\
\hline \hline $\mathrm{T} 2$ & 0.01 & $(1,72,31)$ & 0.094 & 0.085 \\
\hline $\mathrm{T} 3$ & 0.04 & $(1,26,20)$ & 0.234 & 0.231 \\
\hline $\mathrm{T} 4$ & 0.1 & $(1,14,12)$ & 0.431 & 0.462 \\
$\mathrm{~T} 1$ & 0.2 & $(1,11,10)$ & 0.545 & 0.616 \\
\hline $\mathrm{T} 10$ & 0.2 & $(1,12,8)$ & 0.542 & 0.564 \\
\hline $\mathrm{T} 5$ & 0.4 & $(1,11,6)$ & 0.624 & 0.667 \\
\hline $\mathrm{T} 6$ & 0.6 & $(1,7,6)$ & 0.881 & 0.923 \\
\hline $\mathrm{T} 7$ & 1 & $(1,7,4)$ & 0.966 & 0.898 \\
\hline
\end{tabular}

According to Fig. 12a, the (maximum) wave amplitude increases linearly with Rossby number within $0.01 \leq R_{0} \leq 0.2$, and reaches a maximum at $R o=0.4$; afterwards at $0.6 \leq R o \leq 1$, the amplitude drops to a roughly constant value.

Since SIWs are manifested by the vertical velocity, we use the quadratic vertical velocity $\left(W^{2}\right)$ to indicate the energy of SIWs; then we define the ratio $Q=W_{\text {wave }}^{2} / W_{\text {vortex }}^{2}$ to estimate the overall wave intensity, where $W_{\text {wave }}^{2}$ denotes the square of vertical velocity integrated over the volume of SIWs, and $W_{\text {vortex }}^{2}$ represents the square of vertical velocity integrated over the volume of vortices; hereafter, the boundary of vortices is estimated by the zero vorticity contour. In agreement with the wave amplitude, the wave intensity is the largest at $R o=0.4$ (Fig. 12b).

\subsection{Source of SIWs}

Lighthill (1952) conducted a pioneering work on the generation mechanism of sound waves by vortical flows with small Mach number, showing that the unstratified, three-dimensional, unsteady vortical flows will spontaneously generate sound waves. Later, Ford (1994a) extended Lighthill's theory into the $f$-plane, shallow-water equations, and demonstrated that rotating, stratified vortical flows with small Froude number can spontaneously emit 
inertia-gravity waves.

Let's begin with the governing equations (6) for homogeneous, inviscid, rotating fluids:

$$
\begin{aligned}
& \frac{\partial u}{\partial t}+u \frac{\partial u}{\partial x}+v \frac{\partial u}{\partial y}+w \frac{\partial u}{\partial z}-f v=-\frac{1}{\rho} \frac{\partial p}{\partial x} \\
& \frac{\partial v}{\partial t}+u \frac{\partial v}{\partial x}+v \frac{\partial v}{\partial y}+w \frac{\partial v}{\partial z}+f u=-\frac{1}{\rho} \frac{\partial p}{\partial y} \\
& \frac{\partial w}{\partial t}+u \frac{\partial w}{\partial x}+v \frac{\partial w}{\partial y}+w \frac{\partial w}{\partial z}=-\frac{1}{\rho} \frac{\partial p}{\partial z}, \\
& \frac{\partial u}{\partial x}+\frac{\partial v}{\partial y}+\frac{\partial w}{\partial z}=0,
\end{aligned}
$$

where $(u, v, w)$ is the velocity component in $(x, y, z)$ direction; $f$ is the Coriolis parameter; $p$ is the pressure and $\rho$ is the constant density.

After some manipulations (Appendix A), we extend the Lighthill-Ford radiation of inertia-gravity waves into the emission of inertial waves, whose equation is

$$
\frac{\partial^{2}}{\partial t^{2}}\left(\frac{\partial^{2} w}{\partial x^{2}}+\frac{\partial^{2} w}{\partial y^{2}}+\frac{\partial^{2} w}{\partial z^{2}}\right)+f^{2} \frac{\partial^{2} w}{\partial z^{2}}=S
$$

where

$$
S=\sum_{i=1}^{2} \sum_{j=1}^{3} \frac{\partial^{2}}{\partial x_{i} \partial x_{j}} T_{i j}
$$

and

$$
T_{i j}=\frac{\partial}{\partial t}\left[\frac{\partial}{\partial x_{3}}\left(u_{i} u_{j}\right)\right]+f \sum_{m=1}^{2} \frac{\partial}{\partial x_{3}}\left(\varepsilon_{i m} u_{m} u_{j}\right)-\frac{\partial}{\partial t}\left[\sum_{n=1}^{3} \frac{\partial}{\partial x_{n}}\left(\delta_{i j} u_{n} u_{3}\right)\right]
$$

with $\left(x_{1}, x_{2}, x_{3}\right)$ denoting $(x, y, z)$, and $\left(u_{1}, u_{2}, u_{3}\right)$ meaning $(u, v, w) ; \varepsilon_{11}=\varepsilon_{22}=0, \varepsilon_{12}=$ $-\varepsilon_{21}=1 ; \delta_{i j}=1$ (if $i=j$ ) and $\delta_{i j}=0$ (if $i \neq j$ ). The left-hand side of Eq. (7) is the operator of inertial waves, and the right-hand side $S$ represents the wave source that consists of nonlinear terms, including the back-reaction of waves.

Now we use the two contrasting Exps. T1 and C1 to illustrate the function of the wave source $S$. At the early stage of vortex evolution, wave sources in Exps. T1 and C1 both 
have regions of large-magnitude sources $[S \gg \mathcal{O}(1)]$ inside the vortex; these regions exhibit a quadrupole patten (Figs. 13a,b). At the final evolution stage, the vortices in Exp. T1 still owns large-magnitude wave sources (Fig. 13c); nevertheless, the magnitude of wave source in Exp. C1 drops to a level much smaller than $\mathcal{O}(1)$ (Fig. 13d). Recall the evolutions of inertial waves in Exps. T1 and C1. At the early stage, inertial waves are emitted in both Exps. T1 and C1 (Figs. 4b and 9b); at the final stage, inertial waves still exist in Exp. T1 (Fig. 4f), but vanishes in Exp. C1 (Fig. 9d). Based on the corresponding changes between wave sources and wave emissions, we suggest that the wave source $S$ can function as an indicator for the emission of inertial waves. Specifically, if the vortical flow possesses large-magnitude wave sources $[S \gg \mathcal{O}(1)]$, it can emit significant inertial waves, provided that the peak frequency of wave source satisfies the condition to be discussed below. (Note that here $S$ is dimensionless, since the equations (1) and (2) integrated by the model are dimensionless.)

Also, we calculate the wave sources of other target experiments at the final stage of evolution. In terms of Fig. 14, all the wave sources have regions of $S \geq \mathcal{O}(1)$ inside the vortices; consequently, inertial waves are observed in all these experiments. Among the four experiments shown in Fig. 14, the magnitude of $S$ in Exp. T2 (Fig. 14b; Ro = 0.01) is the smallest, only a little over $\mathcal{O}(1)$, which we suggest is the threshold for significant wave emission; accordingly, the wave emission in Exp. T2 is the weakest, with the wave amplitude being several orders of magnitude less than that in other target experiments T3-T7 (cf. Fig. 11).

According to Fig. 15, the most energetic wave frequency listed in Table 2 matches well with the highest peak frequency of wave source; thus, via resonance only these wave modes can gain the most energy from sources. By contrast, for Exp. T8 shown in Fig. 15f, the peak frequency is much larger than 2 , which is the (dimensionless) maximum frequency of inertial waves (cf. Eq. 4). In fact, all peaks are beyond the frequency of 2 ; hence, no inertial waves emit from the wave source in Exp. T8, even though the wave source has $S \gg \mathcal{O}(1)$. 
To sum up, if the wave source has $S \gg \mathcal{O}(1)$ and owns a peak frequency below 2 , significant emissions of inertial waves are expected.

Further, we divide the wave source $S$ in Eq. (8) into three parts based on the three terms of Eq. (9), i.e. $S=S_{1}+S_{2}+S_{3}$ with

$$
\begin{aligned}
& S_{1}=\sum_{i=1}^{2} \sum_{j=1}^{3} \frac{\partial^{2}}{\partial x_{i} \partial x_{j}}\left\{\frac{\partial}{\partial t}\left[\frac{\partial}{\partial x_{3}}\left(u_{i} u_{j}\right)\right]\right\}, \\
& S_{2}=\sum_{i=1}^{2} \sum_{j=1}^{3} \frac{\partial^{2}}{\partial x_{i} \partial x_{j}}\left\{f \sum_{m=1}^{2} \frac{\partial}{\partial x_{3}}\left(\varepsilon_{i m} u_{m} u_{j}\right)\right\}, \\
& S_{3}=\sum_{i=1}^{2} \sum_{j=1}^{3} \frac{\partial^{2}}{\partial x_{i} \partial x_{j}}\left\{-\frac{\partial}{\partial t}\left[\sum_{n=1}^{3} \frac{\partial}{\partial x_{n}}\left(\delta_{i j} u_{n} u_{3}\right)\right]\right\} .
\end{aligned}
$$

Then, we integrate $\left|S_{1}\right|,\left|S_{2}\right|$ and $\left|S_{3}\right|$ over the volume of vortices to estimate the overall intensity of each source term. According to Fig. 16, $S_{1}$ is always smaller than $S_{2}$ and $S_{3}$ but not negligible, and is almost constant at $R o \geq 0.2$. When $R o<0.4$ or so, $S_{2}$ is the primary source; however, the primary source shifts to $S_{3}$ when $R o \geq 0.4$. The total source $S$ increases with Rossby number, and reaches its maximum at $R o=0.2$; then, it drops to a nearly constant value within $0.6 \leq R o \leq 1$

Fig. 16 and Fig. 12 imply that the largest source intensity (at $R o=0.2$ ) does not guarantee the strongest wave intensity (at $R o=0.4$ ), though a positive correlation exists between the wave intensity and source intensity within $0.01 \leq R o \leq 0.2$. Besides, within $0.6 \leq R o \leq 1$, the source intensity remains nearly constant; meanwhile, the wave intensity varies little, too.

\subsection{Effects of SIWs on material dispersion}

Using passive tracers, we investigate the effect of SIWs on material dispersion. Initially, the tracer field $C$ is uniform in the horizontal direction and increases linearly from the top $(z=1)$ to the bottom $(z=0)$, i.e. $C(z)=1-z$. After SIWs being emitted, the tracer 
outside the vortices is organized into spiral stripes (Fig. 17a) that have a pattern similar to SIWs. These stripes increase the horizontal gradients of tracer field, and thus can benefit material diffusion. However, in Exp. C1 no spiral stripes form (Fig. 17b), for no SIWs exist.

Moreover, we compute the vertical advection of tracer, which is $w \frac{\partial C}{\partial z}$ with $w$ and $C$ denoting the vertical velocity and tracer field, respectively. As shown in Fig. 18, lots of spiral stripes characterized by the large-magnitude $w \frac{\partial C}{\partial z}$ emerge in the wave field; the length scales of stripes are visually consistent with that of SIWs. The magnitude of $w \frac{\partial C}{\partial z}$ in Exp. T7 $\left(R_{0}=1\right)$ is larger than that in Exp. $\mathrm{T} 1\left(R_{0}=0.2\right)$ due to the stronger wave intensity in Exp. T7 (cf. Fig. 12). However, no such features appear in Exp. C1, in which SIWs are absent. In the wave field, $w \frac{\partial C}{\partial z}$ is associated with the up and down motions of SIWs; thus according to Eq. (2), SIWs can modify the tracer's local rate of change $\left(\frac{\partial C}{\partial t}\right)$ by advecting tracer vertically, instead of by mixing tracer directly, for the direct tracer mixing occurs at the moment of SIWs breaking. Besides, stronger wave intensities modify the rate more.

Also, Fig. 18 shows that in the same background flow but with different tracer diffusivities $\left(P e=10^{4}, 10^{5}\right)$, the $w \frac{\partial C}{\partial z}$ has similar patterns and magnitudes. This result indicates that the vertical tracer advection by SIWs is insensitive to the tracer diffusivity $(P e)$; at least, there is no significant difference in $w \frac{\partial C}{\partial z}$ between $P e=10^{4}$ and $P e=10^{5}$.

In addition to tracers, we adopt Lagrangian particles to investigate whether SIWs can produce a net vertical transport. In Exp. T7, for example, we release three groups of particles $(\mathrm{A}, \mathrm{B}, \mathrm{C})$ centered on $(2.5,0,0.5),(2.5,2.5,0.5),(0,2.5,0.5)$, respectively (Fig. 19a), at the final stage of evolution; then we monitor the evolution of particles' absolute dispersion in the vertical direction, lasting for two eddy-turnover times. This vertical absolute dispersion is defined by

$$
A_{z}^{2}\left(t, t_{0}\right)=\frac{1}{M} \sum_{i=1}^{M}\left|z_{i}(t)-z_{i}\left(t_{0}\right)\right|^{2}
$$


where $M$ is the number of Lagrangian particles, $z_{i}$ is the z-coordinate of the $i$-th particle; $A_{z}$ quantifies the average vertical distance covered by particles. As shown in Figs. 19b,c,d, the evolutions of vertical absolute dispersion show oscillations, but the minimum dispersion remains almost unchanged (equal to zero); that is, during the two eddy-turnover times, particles move up and down due to SIWs, but do not produce a net vertical transport.

\section{Summary and discussion}

Using numerical simulations of geophysical vortices under background rotation, we have discovered a special class of inertial waves, i.e. spiral inertial waves (SIWs), emitted in a spiral manner from a set of unsteady, non-axisymmetric geophysical vortices; this set of vortices is generated by a single unstable vortex. In particular, the generation of waves requires the vortices to be unsteady, and the formation of spiral patterns needs the vortices to be non-axisymmetric. In addition, we extend the Lighthill-Ford radiation from inertia-gravity waves to inertial waves, and propose an indicator for the emission of inertial waves.

The question whether these emitted spiral waves in the target experiments are pure inertial waves may be raised. Previous studies showed that if the fluid is stratified, vortex Rossby waves can excite inertia-gravity waves (Schecter and Montgomery 2004, 2006; Schecter 2008), which can also create spirals similar to those shown in Fig. 4d. But in the present study, we apply conditions of homogeneous density, rigid lid and flat bottom; thus, gravity waves are ruled out. Besides, vortex Rossby waves diminish outside of the vortices, as the mean potential-vorticity gradients vanish there ( $c f$. Figs. 4a,c,e). In fact, there is a so-called stagnation radius, beyond which no vortex Rossby waves exist (Montgomery and Kallenbach 1997; Moon and Nolan 2010). The stagnation radius implies that vortex Rossby waves are restricted to the near-vortex region, rather than being emitted to the far field. Therefore, these spiral waves emitted into the far field are pure inertial waves. 
In terms of Table 2, when Rossby number decreases, the wavenumber increases; we interpret this relation using the theory of wave capture (Bühler and McIntyre 2005). One result of this theory states that in a background flow with horizontal strain rate $\alpha$, the wavenumber of a wave packet increases rapidly like $\exp (|\alpha| t)$. Thus, we would expect that for a finite time, the wavenumber is larger if the horizontal strain rate $\alpha$ is bigger. Now we examine the square of horizontal strain rate defined by $\alpha^{2}=\left[\left(u_{x}-v_{y}\right)^{2}+\left(v_{x}+u_{y}\right)^{2}\right]$. As is shown in Fig. 20, the magnitude of horizontal strain rate increases while Rossby number decreases; the regions of large strain rate locate at the ends of major axis of the elliptic vortex core. According to Fig. 14, these regions are also the leading wave sources; hence, the wavenumber of inertial waves emitted from these regions, in view of the wave capture theory, increases when Rossby number decreases.

The linear relation between SIWs amplitude and Rossby number within [0.01,0.2] (cf. Fig. 12a) was also reported by Williams et al. (2008), who observed inertia-gravity waves in a rotating, two-layer annulus laboratory experiment. Theoretically, at $R o \ll 1$, ageostrophic effects can be expressed as the first order of $R o$ in an asymptotic expansion of governing equations (Pedlosky 1987). Therefore, we might expect that the amplitudes of ageostrophic motions, like inertial waves and inertia-gravity waves, are scaled as $R o^{\gamma}$ with $\gamma \geq 1$ (Williams et al. 2008); $\gamma=1$ recovers the relation of linearity with Rossby number. Besides the algebraic scaling, exponential scalings were also derived for inertia-gravity waves generated through the breakdown of geostrophic balance, for instance $R o^{-1 / 2} \exp (-\alpha / R o$ ) (Vanneste and Yavneh 2004). However, whether the exponential scalings are applicable to inertial waves needs a farther study.

We find a local maximum of inertial wave emission at $R o=0.4$ ( $c f$. Fig. 12a); likewise, Sugimoto et al. (2008) also report a local maximum of inertia-gravity wave radiation at a moderate Rossby number, and suggested that the local maximum is caused by the shift of primary source. Indeed, as shown in Fig. 16, there is a shift of primary source between 
$S_{2}$ and $S_{3}$ around $R o=0.4$, where the maximum wave amplitude is observed. Therefore, our results confirm that the shift of primary source can amplify the wave emission. From another point of view, the maximum of wave emission at a moderate Rossby number is the result of competition between the dual effects of background rotation on wave emission. That is, at smaller Rossby number, the strong background rotation tends to suppress the vertical motions and thereby the wave emission (Ford et al. 2000); for instance, the wave emission is the weakest in Exp. T2 $\left(R_{0}=0.01\right)$. Nevertheless, at larger Rossby number, since inertial waves arise thanks to the Coriolis force, the weak background rotation (weak Coriolis force) damages the emission of inertial waves; for example, no inertial waves are observed in Exp. T8 $(R o=5)$.

The theory of Lighthill-Ford radiation was tested by laboratory experiments of inertia-gravity wave radiation (e.g. Williams et al. 2005), and was adopted into practical applications, for example the forecast of clear-air turbulence (Knox et al. 2008). By extending the Lighthill-Ford radiation into inertial waves, we derive a wave source $S$ given by Eq. (8) or Eq. (10). Since the source $S$ does not include the dissipation due to fluid viscosity, the condition of $S \neq 0$ is necessary but not sufficient for the emission of inertial waves in viscous fluids like the ocean and atmosphere; in other words, the dissipation will weaken and may even eliminate inertial waves, particularly for $S \ll \mathcal{O}(1)$.

In addition to a wave source with $S \gg \mathcal{O}(1)$, the emission of inertial waves also requires that the peak frequency of wave source needs to be smaller than the Coriolis parameter $f$, which is the cut-off frequency of inertial waves. Otherwise, even though the wave source has $S \gg \mathcal{O}(1)$, the source still cannot emit inertial waves; for example in Exp. T8, no inertial waves are observed as the peak frequency of wave source is much larger than the cut-off frequency of inertial waves ( $c f$. Fig. 15f). Similarly, in the study of inertia-gravity wave radiation, Sugimoto et al. (2008) and Sugimoto and Ishii (2012) pointed out that an important factor that determines the inertia-gravity wave radiation is the relative relation 
between the peak frequency of wave source and the cut-off frequency of inertia-gravity waves. Therefore, we suggest that if the wave source owns $S \gg \mathcal{O}(1)$ and a peak frequency smaller than the cut-off frequency of inertial waves, the source can emit significant inertial waves that possess large amplitudes; if $S \leq \mathcal{O}(1)$, inertial waves may also be emitted, but are difficult to be detected due to small wave amplitudes.

At the final stage of vortex evolution, SIWs packets are emitted continuously and keep stationary relative to the tripolar vortices; that is, the group velocity of SIWs is equal to the phase speed of tripolar vortices, or equivalently vortex Rossby waves, which control the phase speed of vortices in our experiments. This relation of wave stationarity relative to the vortices was also documented in studies of inertia-gravity waves (e.g. Snyder et al. 2007; Snyder et al. 2009; Viúdez 2007, 2008; Wang et al. 2009). We interpret the relative stationarity based on the theory of interactions between long and short waves (Benney 1977). In the present context, this theory would express that owing to the resonant triad interactions, energy is transferred to SIWs (short waves) from vortex Rossby waves (long waves), if and only if the group velocity of SIWs is equal to the phase speed of vortex Rossby waves. Hence, only the waves that are stationary relative to the vortices can persistently acquire energy.

The asymmetry between cyclone and anticyclone under background rotation was found based on evidences, such as the evolution of vortex (e.g. Kloosterziel and van Heijst 1991), growth rate of vortical flow instabilities (e.g. Kloosterziel et al. 2007; Wang and Özgökmen 2015) and inertia-gravity wave radiation (e.g. Kuo and Polvani 2000; Sugimoto et al. 2015). According to the study of Sugimoto et al. (2015), this asymmetry comes from the source term that originates in the Coriolis force, i.e. the second term of wave source Eq. (9). Physically, the asymmetry is caused by the reverse of direction of Coriolis force between cyclone and anticyclone. Our study enriches the cyclone-anticyclone asymmetry in the subject of inertial wave emission. In Exps. T1 and T10, this asymmetry is elucidated by the wave amplitude and the variation along radius (Figure 21); particularly in the field of radius $>2$, SIWs emitted 
from the anticyclone have a larger amplitude. In fact, the phenomenon of stronger wave radiation from anticyclones was also found in inertia-gravity wave radiation (e.g. Snyder et al. 2007; Viúdez 2007; Wang et al. 2009; Sugimoto et al. 2015). In addition, this asymmetry is also manifested by the difference in magnitudes of wave sources between Exps. T1 and T10 at the final stage of vortex evolution ( $c f$. Fig. 13c and Fig. 14a).

On the material dispersion, although SIWs do not produce a net vertical transport, they can generate tracer filaments ( $c f$. Fig. 17a), which favor the tracer diffusion and therefore mixing. The tracer used in this study can represent materials such as salinity, temperature, dissolved gases, and nitrates; thus, we expect that SIWs can also benefit the mixing of these materials by creating filaments.

According to Fig. 17a, SIWs organize the tracer into spiral stripes, which are similar to some spiral features observed in the ocean and atmosphere, such as spiral sea eddies (e.g. Munk et al. 2000; Ivanov and Ginzburg 2002) and spiral hurricane rainbands (e.g. Willoughby 1978; Gall, Tuttle and Hildebrand 1998). However, the formation mechanisms of these spiral features are controversial. For example, it was argued that the baroclinic or frontal instability is responsible for the development of spiral eddies (Munk et al. 2000; Eldevik and Dysthe 2002); nevertheless, Shen and Evans (2002) interpreted the spiral eddies using inertial instability without density anomaly, which is crucial for the frontal or baroclinic instability. Similar for spiral rainbands, there are also debates on the formation mechanism, like the inertia-gravity wave (e.g. Kurihara 1976; Willoughby 1978) and vortex Rossby wave (e.g. Guinn and Schubert 1993; Montgomery and Kallenbach 1997; Chen and Yau 2001). In this study, based on the similarity between the spirals of SIWs and the observed spiral features, we suggest that SIWs offer another way to generate these spiral eddies and rainbands. However, our experiments exclude the density anomaly, which may cause gaps between numerical experiments and the reality. On the other hand, our results imply that the density anomaly is not necessary to generate spiral features; further, we infer that the 
baroclinic or frontal instability is unlikely to play a key role in the formation of spiral features.

Last, we argue that the parameterization of inertial waves need to be considered in general circulation models, particularly in modelling well-mixed flows. As the parameter regimes $(R o, R e)$ used in our numerical experiments are relevant to real geophysical flows, the indicator $S$ for the emission of inertial waves can be applied into the general circulation models. A possible procedures is as follows. Compute the wave source at each time step, and check whether the magnitude of wave source is beyond the threshold and whether the peak frequency of wave source is below the cut-off frequency. Then activate the parameterization only in regions where all the aforementioned conditions are satisfied.

\section{Acknowledgements}

P. Wang and T. Özgökmen acknowledge the support from the Office of Naval Research (grant \#N000141110087). T. Özgökmen's effort is also partially funded by a grant from the $\mathrm{BP} /$ The Gulf of Mexico Research Initiative. The authors thank two anonymous reviewers for their insightful comments and constructive suggestions.

\section{Appendix A Extension of Lighthill-Ford radiation into}

\section{inertial waves}

The flux form of Eqs. (6) is:

$$
\begin{aligned}
& \frac{\partial u}{\partial t}-f v+\frac{\partial P}{\partial x}=-\left(\frac{\partial u u}{\partial x}+\frac{\partial u v}{\partial y}+\frac{\partial u w}{\partial z}\right) \\
& \frac{\partial v}{\partial t}+f u+\frac{\partial P}{\partial y}=-\left(\frac{\partial u v}{\partial x}+\frac{\partial v v}{\partial y}+\frac{\partial v w}{\partial z}\right), \\
& \frac{\partial w}{\partial t}+\frac{\partial P}{\partial z}=-\left(\frac{\partial u w}{\partial x}+\frac{\partial v w}{\partial y}+\frac{\partial w w}{\partial z}\right)
\end{aligned}
$$


where $P=p / \rho$. Take $\frac{\partial(\mathrm{A} .1 \mathrm{~b})}{\partial x}-\frac{\partial(\mathrm{A} .1 \mathrm{a})}{\partial y}$, obtaining

$$
\frac{\partial \zeta}{\partial t}=f \frac{\partial w}{\partial z}-\left(\frac{\partial^{2} u v}{\partial x^{2}}+\frac{\partial^{2} v v}{\partial x \partial y}+\frac{\partial^{2} v w}{\partial x \partial z}\right)+\left(\frac{\partial^{2} u u}{\partial x \partial y}+\frac{\partial^{2} u v}{\partial y^{2}}+\frac{\partial^{2} u w}{\partial y \partial z}\right)
$$

where $\zeta=\frac{\partial v}{\partial x}-\frac{\partial u}{\partial y}$. Take $\frac{\partial}{\partial t}\left[\frac{\partial(\mathrm{A} \cdot 1 \mathrm{a})}{\partial x}+\frac{\partial(\mathrm{A} .1 \mathrm{~b})}{\partial y}\right]$, obtaining

$$
\begin{aligned}
& \frac{\partial^{2}}{\partial t^{2}}\left(\frac{\partial w}{\partial z}\right)+f \frac{\partial \zeta}{\partial t}-\frac{\partial}{\partial t}\left(\frac{\partial^{2} P}{\partial x^{2}}+\frac{\partial^{2} P}{\partial y^{2}}\right)= \\
& \frac{\partial}{\partial t}\left(\frac{\partial^{2} u u}{\partial x^{2}}+\frac{\partial^{2} u v}{\partial x \partial y}+\frac{\partial^{2} u w}{\partial x \partial z}\right)+\frac{\partial}{\partial t}\left(\frac{\partial^{2} u v}{\partial x \partial y}+\frac{\partial^{2} v v}{\partial y^{2}}+\frac{\partial^{2} v w}{\partial y \partial z}\right)
\end{aligned}
$$

Substitute Eq. (A.2) into Eq. (A.3) and then take $\frac{\partial}{\partial z}$, obtaining

$$
\begin{aligned}
& -\frac{\partial}{\partial t}\left(\frac{\partial^{2}}{\partial x^{2}}+\frac{\partial^{2}}{\partial y^{2}}\right) \frac{\partial P}{\partial z}+\frac{\partial^{2}}{\partial t^{2}}\left(\frac{\partial^{2} w}{\partial z^{2}}\right)+f^{2} \frac{\partial^{2} w}{\partial z^{2}}= \\
& \frac{\partial^{2}}{\partial t \partial z}\left[\left(\frac{\partial^{2} u u}{\partial x^{2}}+\frac{\partial^{2} u v}{\partial x \partial y}+\frac{\partial^{2} u w}{\partial x \partial z}\right)+\left(\frac{\partial^{2} u v}{\partial x \partial y}+\frac{\partial^{2} v v}{\partial y^{2}}+\frac{\partial^{2} v w}{\partial y \partial z}\right)\right]+ \\
& f \frac{\partial}{\partial z}\left[\left(\frac{\partial^{2} u v}{\partial x^{2}}+\frac{\partial^{2} v v}{\partial x \partial y}+\frac{\partial^{2} v w}{\partial x \partial z}\right)-\left(\frac{\partial^{2} u u}{\partial x \partial y}+\frac{\partial^{2} u v}{\partial y^{2}}+\frac{\partial^{2} u w}{\partial y \partial z}\right)\right]
\end{aligned}
$$

Last, substitute Eq. (A.1c) into Eq. (A.4) to eliminate $\frac{\partial P}{\partial z}$, yielding Eqs. (7-9) for inertial waves.

\section{References}

[1] Aldridge, K. D., Lumb, L. I., 1987. Inertial waves identified in the Earth's fluid outer core. Nature 325, 421-423.

[2] Benney, D. J., 1977. A general theory for interactions between short and long waves. Stud. Appl. Maths. 56, 81-94.

[3] Boisson, J., Lamriben, C., Maas, L. R. M., Cortet, P., Moisy, F., 2012. Inertial waves and modes excited by the libration of a rotating cube. Phys. Fluid 24, 076602. 
[4] Bordes, G. Moisy, F., Dauxois, T., Cortet, P., 2012. Experimental evidence of a triadic resonance of plane inertial waves in a rotating fluid. Phys. Fluid 24, 014105.

[5] Bühler, O., McIntyre, M. E., 2005. Wave capture and wave-vortex duality. J. Fluid Mech. 534, 67-95.

[6] Chabreyrie, R., Llewellyn Smith, S. G., 2014. Short- and long-time transport structures in a three dimensional time dependent flow. arXiv:1405.2022.

[7] Chen, Y., Yau, M. K., 2001. Spiral bands in a simulated hurricane. Part I: vortex Rossby wave verification. J. Atmos. Sci. 58, 2128-2145.

[8] Cortet, P., Lamriben, C., Moisy, F., 2010. Viscous spreading of an inertial wave beam in a rotating fluid. Phys. Fluid 22, 086603.

[9] Eldevik, T., Dysthe, K. B., 2002. Spiral eddies. J. Phys. Oceano. 32,851-869.

[10] Fischer, P. F., 1997. An overlapping Schwarz method for spectral element solution of the incompressible Navier-Stokes equations. J. Comp. Phys. 133, 84-101.

[11] Ford, R., 1994a. Gravity wave radiation from vortex trains in rotating shallow water. J. Fluid Mech. 281, 81-118.

[12] Ford, R., 1994b. The response of a rotating ellipse of uniform potential vorticity to gravity wave radiation. Phys. Fluid 6, 3694 .

[13] Ford, R., McIntyre, M. E., Norton, W. A., 2000. Balance and the slow quasimanifold: some explicit results. J. Atmos. Sci. 57, 1236-1254.

[14] Ford, R., McIntyre, M. E., Norton, W. A., 2002. Reply. J. Atmos. Sci. 59, 2878-2882.

[15] Fultz, D., 1959. A note on overstability and the elastoid-inertia oscillations of Kelvin, Solberg, and Bjerknes. J. Meteor. 16, 199-208. 
[16] Gall, R., Tuttle, J., Hildebrand, P., 1998. Small-scale spiral bands observed in hurricanes Andrew, Hugo, and Erin. Mon. Wea. Rev. 126, 1749-1766.

[17] Gill, A. E., 1982. Atmosphere-Ocean Dynamics. Academic Press.

[18] Greenspan, H. P., 1969. The theory of rotating fluids. Cambridge University Press.

[19] Guinn, T. A., Schubert, W. H., 1993. Hurricane spiral bands. J. Atmos. Sci. 50, 3380-3403.

[20] Gula, J., Plougonven, R., Zeitlin, V., 2009. Ageostrophic instabilities of fronts in a channel in a stratified rotating fluid. J. Fluid Mech. 627, 485-507.

[21] Ivanov, A. Y., Ginzburg, A. I., 2002. Oceanic eddies in synthetic aperture radar images. Proc. Indian Acad. Sci. 111, 281-295.

[22] Kelley, D. H., Triana, S. A., Zimmerman, D. S., Tilgner, A., Lathrop, D. P., 2007. Inertial waves driven by differential rotation in a planetary geometry. Geophys. Astrophys. Fluid Dyn. 101, 469-487.

[23] Kelvin, L., 1880. Vibrations of a columnar vortex. Phil. Mag. 10, 155-168.

[24] Kloosterziel, R. C., Carnevale, G. F., Orlandi, P., 2007. Inertial instability in rotating and stratified fluids: barotropic vortices. J. Fluid Mech. 583, 379-412.

[25] Kloosterziel, R. C., van Heijst, G. J. F., 1991. An experimental study of unstable barotropic vortices in a rotating fluid. J. Fluid Mech. 223, 1-24.

[26] Knox, J. A., McCann, D. W., Williams, P. D., 2008. Application of the Lighthill-Ford theory of spontaneous imbalance to clear-air turbulence forecasting. J. Atmos. Sci. 65, $3292-3304$.

[27] Kuo, A. C., Polvani, L. M., 2000. Nonlinear geostrophic adjustment, cyclone/anticyclone asymmetry, and potential vorticity rearrangement. Phys. Fluid 12(5), 1087-1100. 
[28] Kurihara, Y., 1976. On the development of spiral bands in a tropical cyclone. J. Atmos. Sci 33, 940-958.

[29] Ledwell, J. R., McGillicuddy, D. J., Anderson, L. A., 2008. Nutrient flux into an intense deep chlorophyll layer in a mode-water eddy. Deep-Sea Res. II 55, 1139-1160.

[30] Lighthill, M. J., 1952. On sound generated aerodynamically. I. General theory. Proc. R. Soc. Lond. 211, 564-587.

[31] Lopez, J. M., Marques, F., 2011. Instabilities and inertial waves generated in a librating cylinder. J. Fluid Mech. 687, 171-193.

[32] Manasseh, R., 1992. Breakdown regimes of inertia waves in a precessing cylinder. J. Fluid Mech. 243, 261-296.

[33] McEwan, A. D., 1970. Inertial oscillations in a rotating fluid cylinder. J. Fluid Mech. 40, 603-640.

[34] McIntyre, M. E., 2009. Spontaneous imbalance and hybrid vortex-gravity structures. J. Atmos. Sci. 66, 1315-1326.

[35] Messio, L., Morize, C., Rabaud, M., Moisy, F., 2008. Experimental observation using particle image velocimetry of inertial waves in a rotating fluid. Exp. Fluids 44, 519-528.

[36] Molemaker, M. J., McWilliams, J. C., Yavneh, I., 2005. Baroclinic instability and loss of balance. J. Phys. Oceano. 35, 1505-1517.

[37] Montgomery, M. T., Kallenbach, R. J., 1997. A theory for vortex Rossby-waves and its application to spiral bands and intensity changes in hurricanes. Quart. J. Roy. Meteor. Soc. 123, 435-465.

[38] Moon, Y., Nolan, D. S., 2010. Do gravity waves transport angular momentum away from tropical cyclones? J. Atmos. Sci. 67, 117-135. 
[39] Munk, W., Armi, L., Fischer, K., Zachariasen, F., 2000. Spirals on the sea. Proc. R. Soc. Lond. A 456, 1217-1280.

[40] Noir, J., Hemmerlin, F., Wicht, J., Baca, S. M., Aurnou, J. M., 2009. An experimental and numerical study of vibrationally driven flow in planetary cores and subsurface oceans. Phys. Earth Planet. In. 173, 141-152.

[41] Ohlmann, J. C., LaCasce, J. H., Washburn, L., Mariano, A. J., Emery, B., 2012. Relative dispersion observations and trajectory modeling in the Santa Barbara Channel. J. Geophys. Res. 117, C05040.

[42] O'Sullivan, D., Dunkerton, T. J., 1995. Generation of inertia-gravity waves in a simulated life cycle of baroclinic instability. J. Atmos. Sci. 52, 3695-3716.

[43] Özgökmen, T. M., Poje, A. C., Fischer, P. F., Haza, A. C., 2011. Large eddy simulations of mixed of mixed layer instabilities and sampling strategies. Ocean Model. 39, 311-331.

[44] Özgökmen, T. M., Poje, A. C., Fischer, P. F., Childs, H., Krishnan, H., Garth, C., Haza, A. C., Ryan, E., 2012. On multi-scale dispersion under the influence of surface mixed layer instabilities and deep flows. Ocean Model. 56, 16-30.

[45] Patera, A. T., 1984. A spectral element method for fluid dynamics: laminar flow in a channel expansion. J. Comp. Phys. 54, 468-488.

[46] Pedlosky, J., 1987. Geophysical Fluid Dynamics. 2nd ed. Springer-Verlag.

[47] Plougonven, R., Muraki, D. J., Snyder, C., 2005. A baroclinic instability that couples balanced motions and gravity waves. J. Atmos. Sci. 62, 1545-1559.

[48] Plougonven, R., Zhang, F., 2014. Internal gravity waves from atmospheric jets and fronts. Rev. Geophys. 52, 33-76. 
[49] Pratt, L. J., Rypina, I. I., Özgökmen, T. M., Wang, P., Childs, H., Bebieva, Y., 2014. Chaotic advection in a steady, three-dimensional, Ekman-driven eddy. J. Fluid Mech. $738,143-183$.

[50] Rossby, C. G., 1938. On the mutual adjustment of pressure and velocity distributions in certain simple current systems, II. J. Mar. Res. 2, 239-263.

[51] Rypina, I. I., Llopiz, J. K. Pratt, L. J., Lozier, M. S., 2014. Dispersal pathways of American eel larvae from the Sargasso Sea. Limnol. Oceanogr. 59(5), 1704-1714.

[52] Rypina, I. I., Pratt, L. J., Wang, P., Özgökmen, T. M., Mezić, I, 2015. Resonance phenomena in a time-dependent, three-dimensional model of an idealized eddy. Chaos: An Interdisciplinary J. Non. Sci. 25, 087401.

[53] Schecter, D. A., 2008. The spontaneous imbalance of an atmospheric vortex at high Rossby number," J. Atmos. Sci. 65, 2498-2521.

[54] Schecter, D. A., Montgomery, M. T., 2004. Damping and pumping of a vortex Rossby wave in a monotonic cyclone: Critical layer stirring versus inertia-buoyancy wave emission. Phys. Fluid 16, 1334.

[55] Schecter, D. A., Montgomery, M. T., 2006. Conditions that inhibit the spontaneous radiation of spiral inertia-gravity waves from an intense mesoscale cyclone. J. Atmos. Sci. 63, 435-456.

[56] Shen, C. Y., Evans, T. E., 2002. Inertial instability and sea spirals. Geophys. Res. Lett. $29,2124$.

[57] Snyder, C., Muraki, D. J., Plougonven, R., Zhang, F., 2007. Inertia-gravity waves generated within a dipole vortex. J. Atmos. Sci. 64, 4417-4431.

[58] Snyder, C., Plougonven, R., Muraki, D. J., 2009. Mechanisms for spontaneous gravity wave generation within a dipole vortex. J. Atmos. Sci. 66, 3464-3478. 
[59] Sugimoto, N., Ishii, K., 2012. Spontaneous gravity wave radiation in a shallow water system on a rotating sphere. J. Meteor. Soc. Japan 90(1), 101-125.

[60] Sugimoto, N., Ishioka, K., Kobayashi, H., Shimomura, Y., 2015. Cyclone-anticyclone asymmetry in gravity wave radiation from a co-rotating vortex pair in rotating shallow water. J. Fluid Mech. 722, 80-106.

[61] Sugimoto, N., Ishioka, K., Ishii, K., 2008. Parameter sweep experiments on spontaneous gravity wave radiation form unsteady rotational flow in an f-plane shallow water system. J. Atmos. Sci. 65, 235-249.

[62] Sugimoto, N., Ishioka, K., Yoden, S., 2007. Gravity wave radiation from unsteady rotational flow in an f-plane shallow water system. Fluid Dyn. Res. 39, 731-754.

[63] Tilgner, A., 2007. Zonal wind driven by inertial modes. Phys. Rev. Lett. 99, 194501.

[64] Vanneste, J., Yavneh, I., 2004. Exponentially small inertia-gravity waves and the breakdown of quasigeostrophic balance. J. Atmos. Sci. 61, 211-223.

[65] Vanneste, J., 2013. Balance and spontaneous wave generation in geophysical flows. Annu. Rev. Fluid Mech. 45, 147-172.

[66] Viúdez, Á., 2006. Spiral patterns of inertia-gravity waves in geophysical flows. J. Fluid Mech. 562, 73-82.

[67] Viúdez, Á., 2007. The origin of the stationary frontal wave packet spontaneously generated in rotating stratified vortex dipoles. J. Fluid Mech. 593, 359-383.

[68] Viúdez, Á., 2008. The stationary frontal wave packet spontaneously generated in mesoscale dipoles. J. Phys. Oceano. 38, 243-256.

[69] Wang, S., Zhang, F., Snyder, C., 2009. Generation and propagation of inertia-gravity waves from vortex dipoles and jets. J. Atmos. Sci. 66, 1294-1314. 
[70] Wang, S., Zhang, F., 2010. Source of gravity waves within a vortex-dipole jet revealed by a linear model. J. Atmos. Sci. 67, 1438-1455.

[71] Wang, P., Özgökmen, T. M., 2015. How do hydrodynamic instabilities affect 3D transport in geophysical vortices? Ocean Model. 87, 48-66.

[72] Williams, P. D., Haine, T. W. N., Read, P. L., 2005. On the generation mechanisms of short-scale unbalanced modes in rotating two-layer flows with vertical shear. J. Fluid Mech. 528, 1-22.

[73] Williams, P. D., Haine, T. W. N., Read, P. L., 2008. Inertia-gravity waves emitted from balanced flow: observations, properties, consequences. J. Atmos. Sci. 65, 3543-3556.

[74] Willoughby, H. E., 1978. A possible mechanism for the formation of hurricane rainbands. J. Atmos. Sci. 35, 838-848.

[75] Zhang, F., Koch, S. E., Davis, C. A., Kaplan, M. L., 2000. A survey of unbalanced flow diagnostics and their application. Adv. Atmos. Sci. 17, 165-183.

[76] Zhang, F., Koch, S. E., Davis, C. A., Kaplan, M. L., 2001. Wavelet analysis and the governing dynamics of a large-amplitude mesoscale gravity-wave event along the East Coast of the United States. Quart. J. Roy. Meteor. Soc. 127, 2209-2245.

[77] Zhang, F., 2004. Generation of mesoscale gravity waves in upper-tropospheric jet-front systems. J. Atmos. Sci. 61, 440-457. 


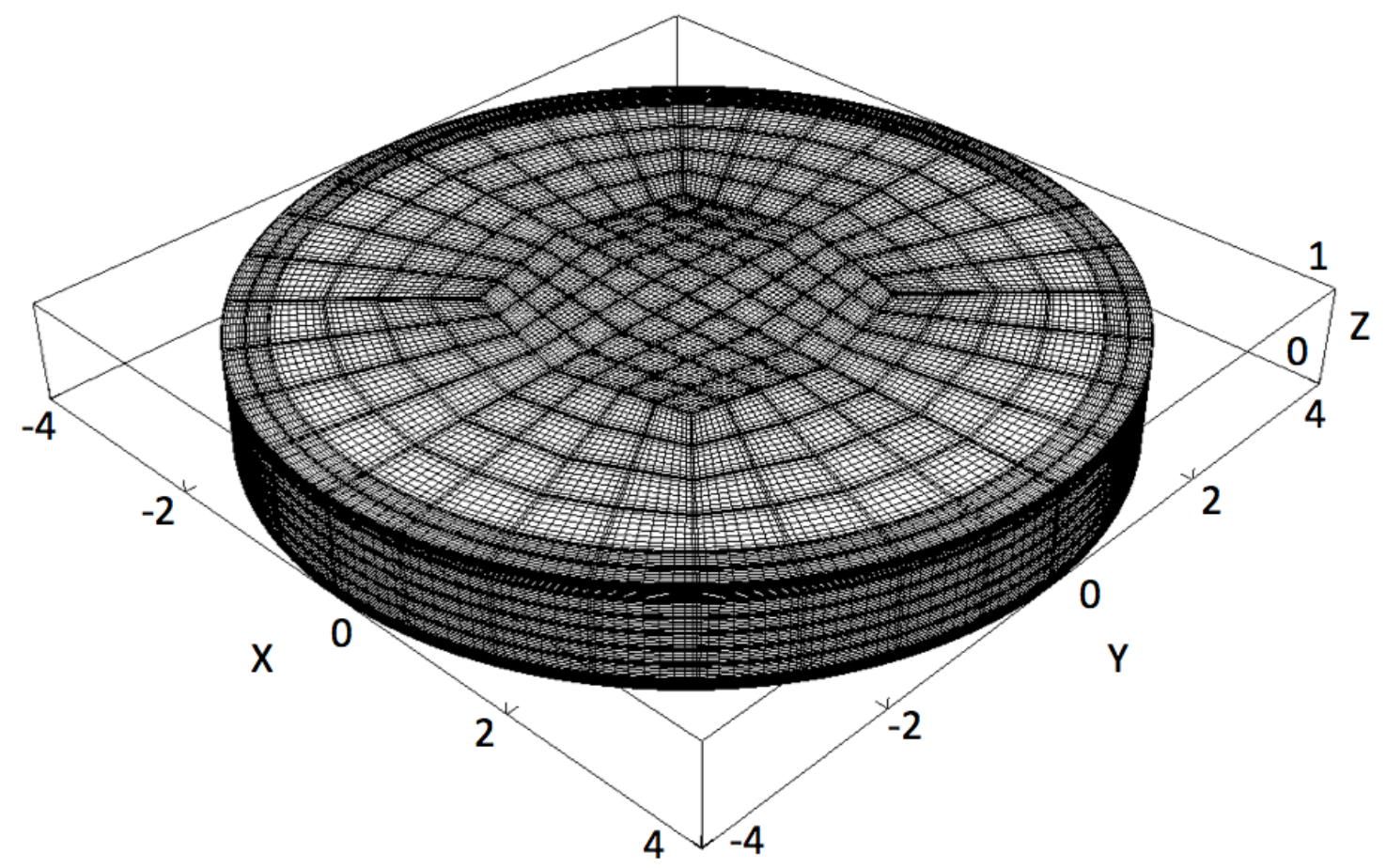

Figure 1: The cylindrical domain used in the numerical experiments. 


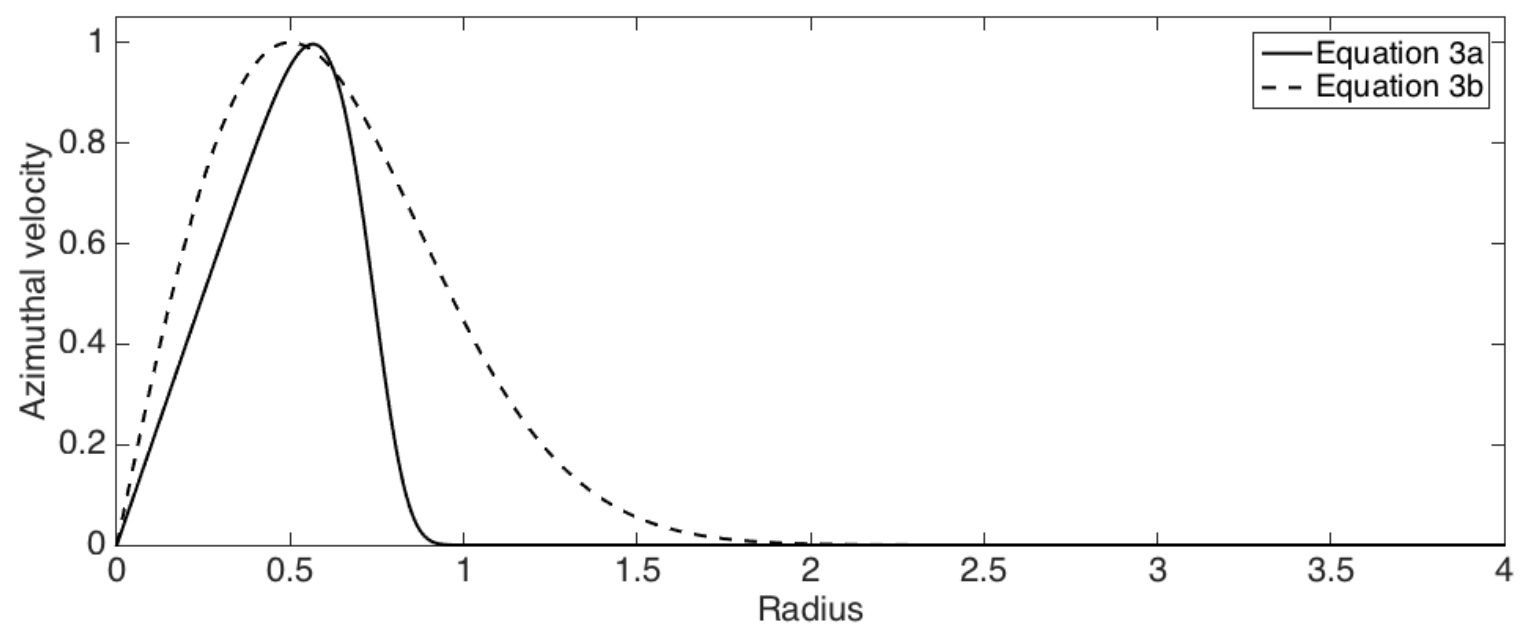

Figure 2: Azimuthal velocity profiles of the initial vortex. 


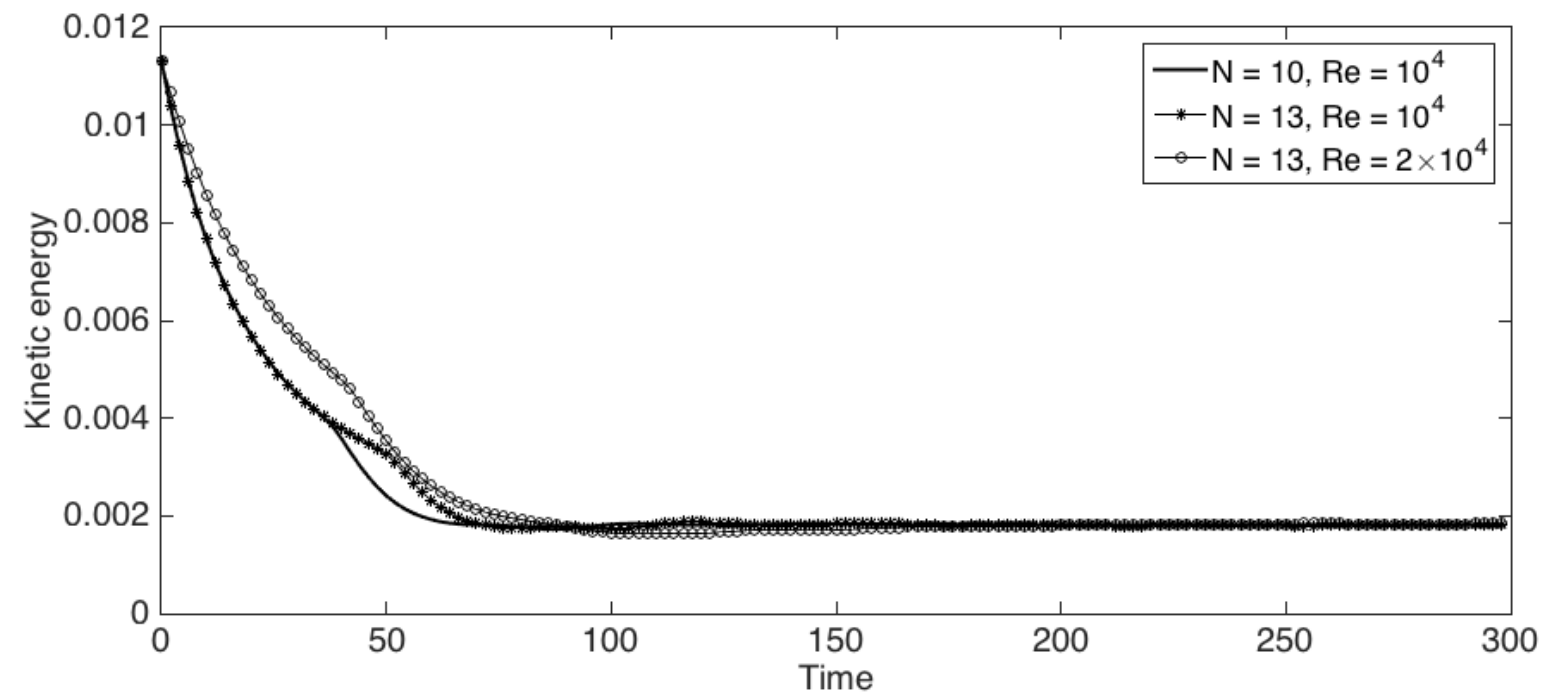

Figure 3: The evolutions of kinetic energy of Exp. T1 with different mesh resolutions and Reynolds numbers. 


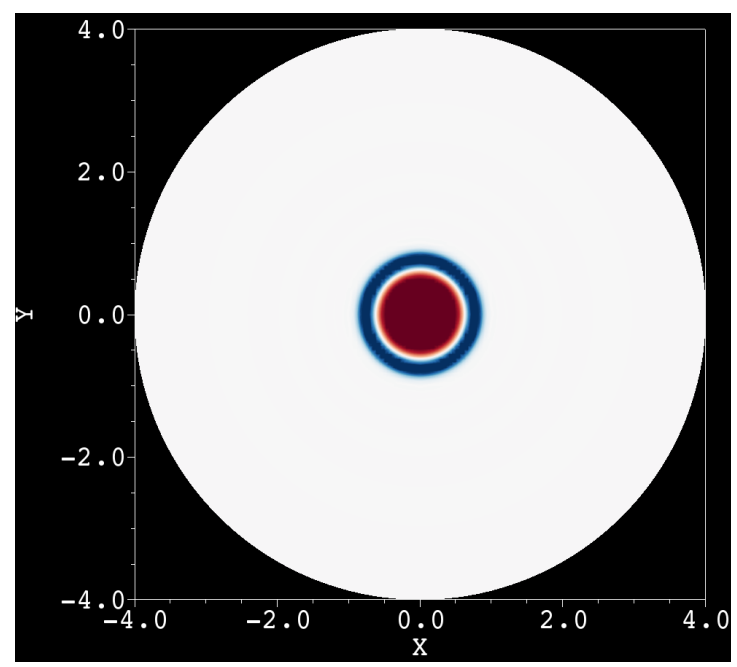

(a)

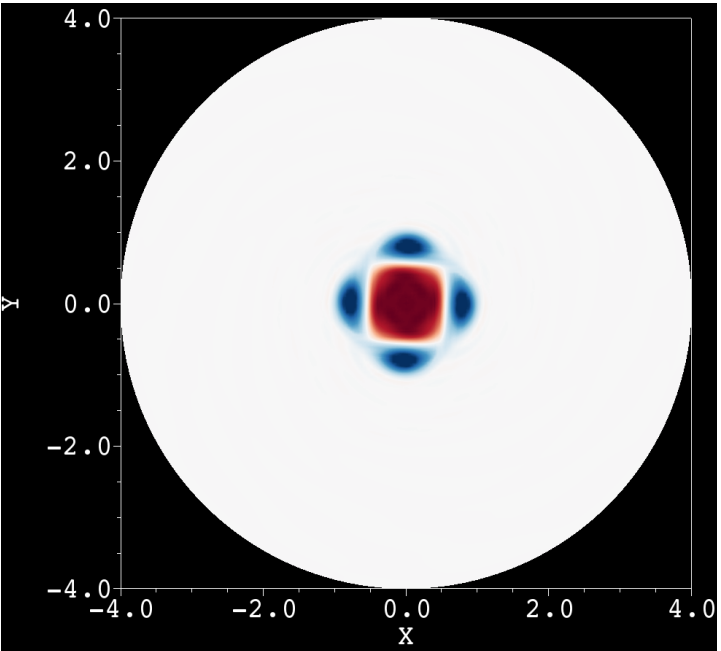

(c)

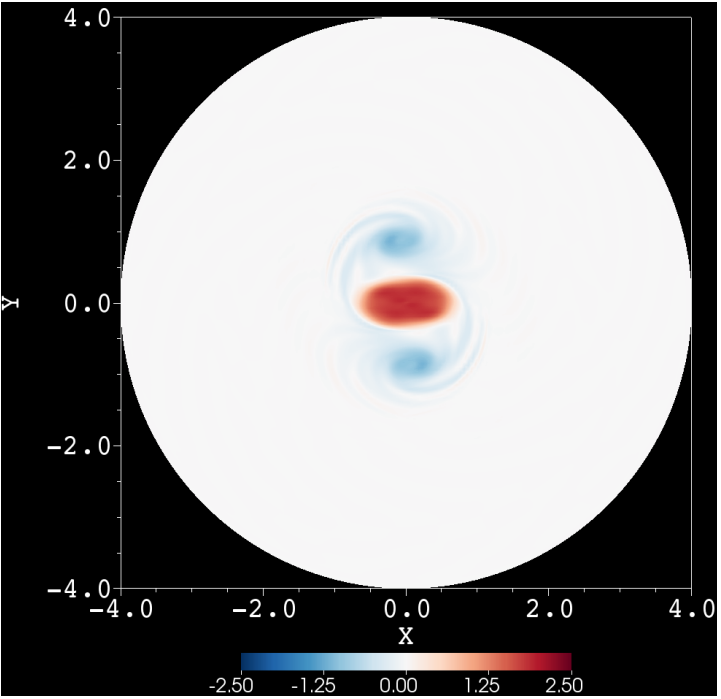

(e)

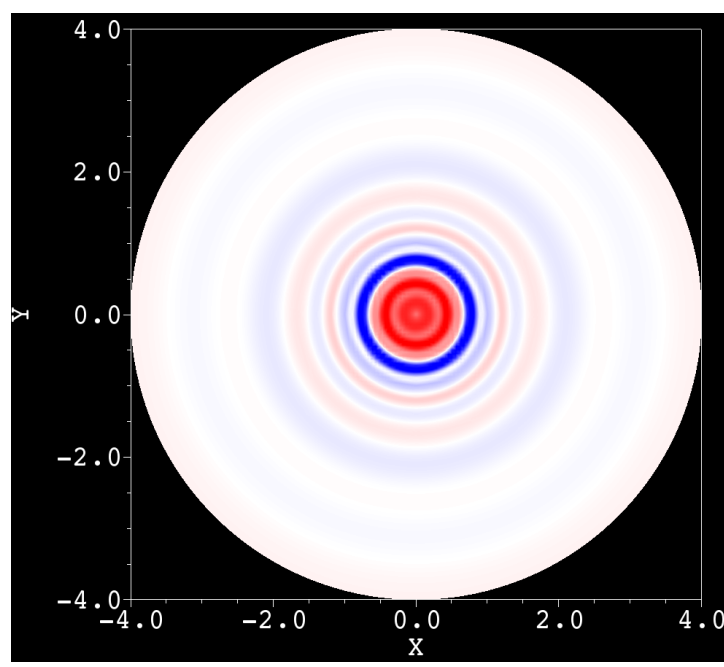

(b)

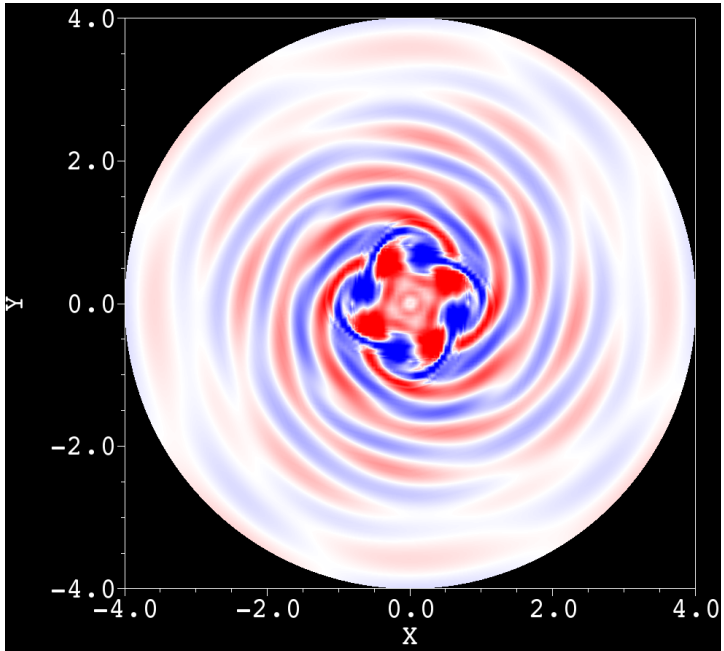

(d)

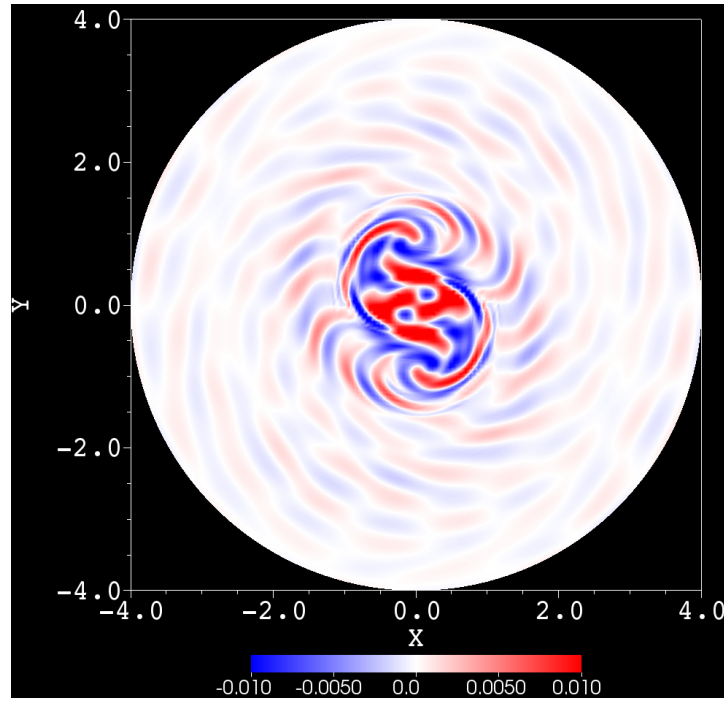

(f)

Figure 4: Vertical vorticity fields (left column) and vertical velocity fields (right column) from Exp. T1 (initially cyclonic); both fields are plotted at mid-depth horizontal plane at (a, b) time $=6 ;(\mathrm{c}, \mathrm{d})$ time $=30$ and $(\mathrm{e}, \mathrm{f})$ time $=204$. (The https ://youtu $\cdot$ be/Vw564T17pk4 links the wave animation.) 


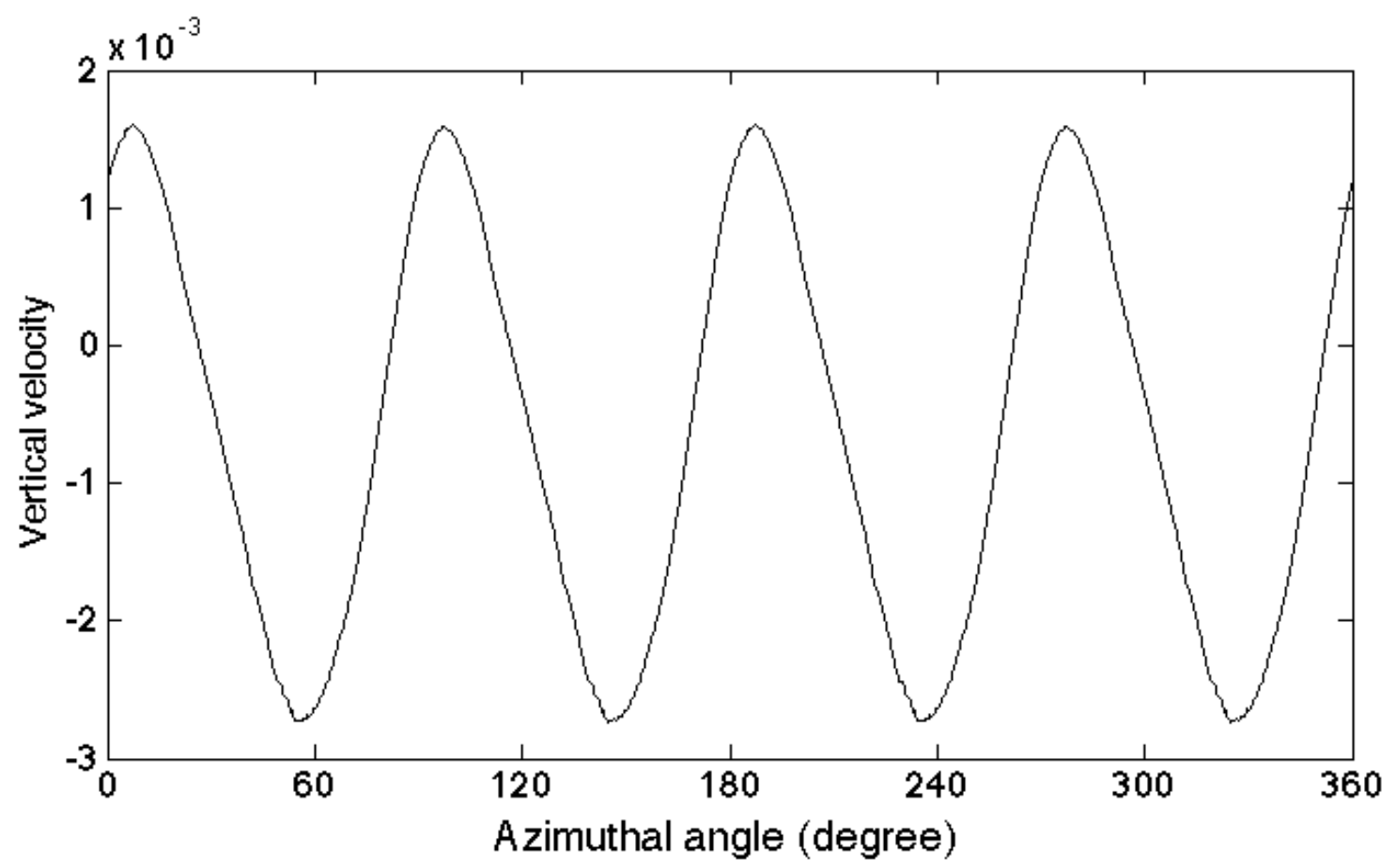

(a)

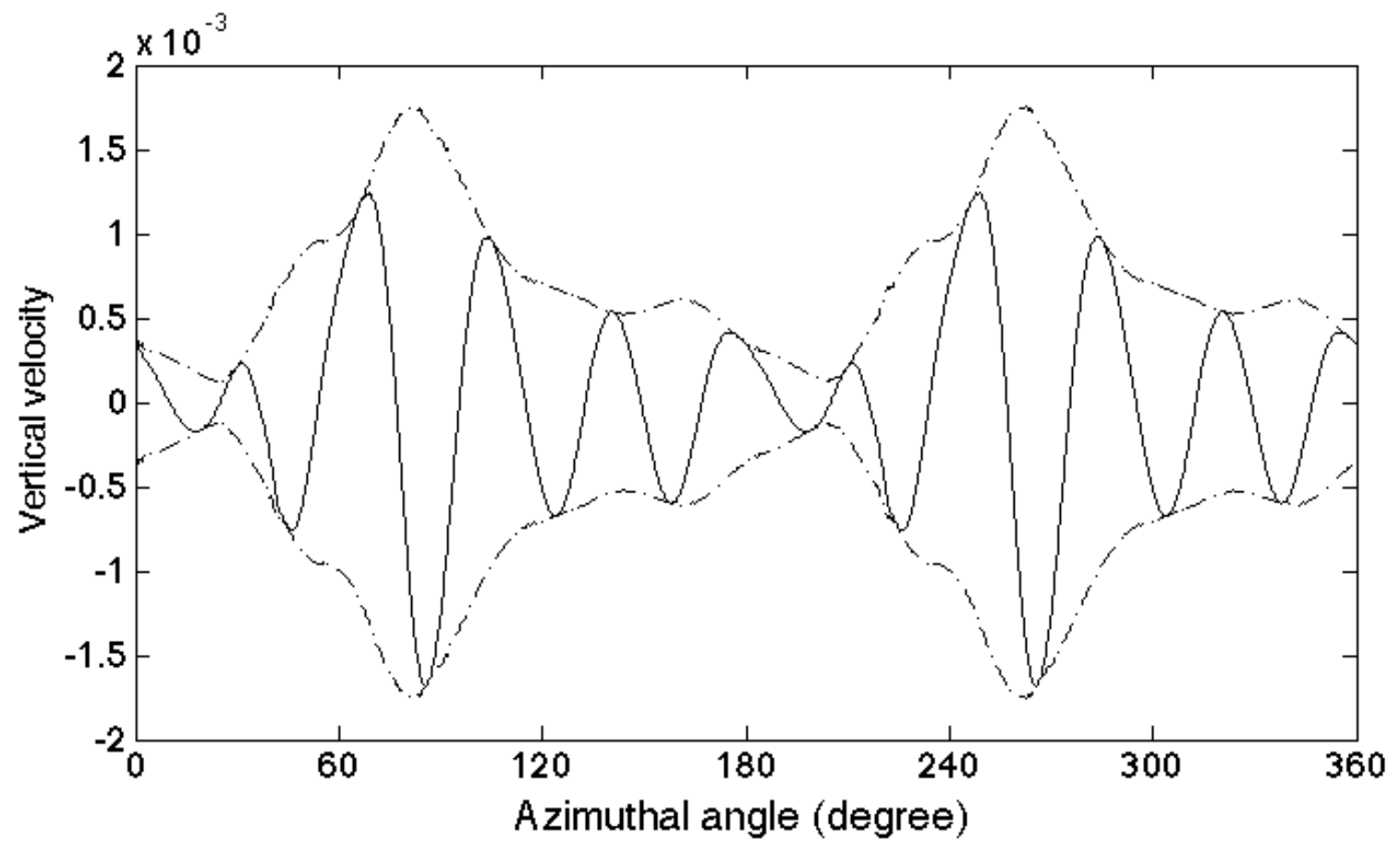

(b)

Figure 5: Vertical velocity along the circle of radius $r=2.5$ at the mid-depth in Exp. T1. (a) SIW-4 phase at time $=30$ and (b) SIW-2 phase at time $=204$ (dashed lines outlining the wave envelope). 


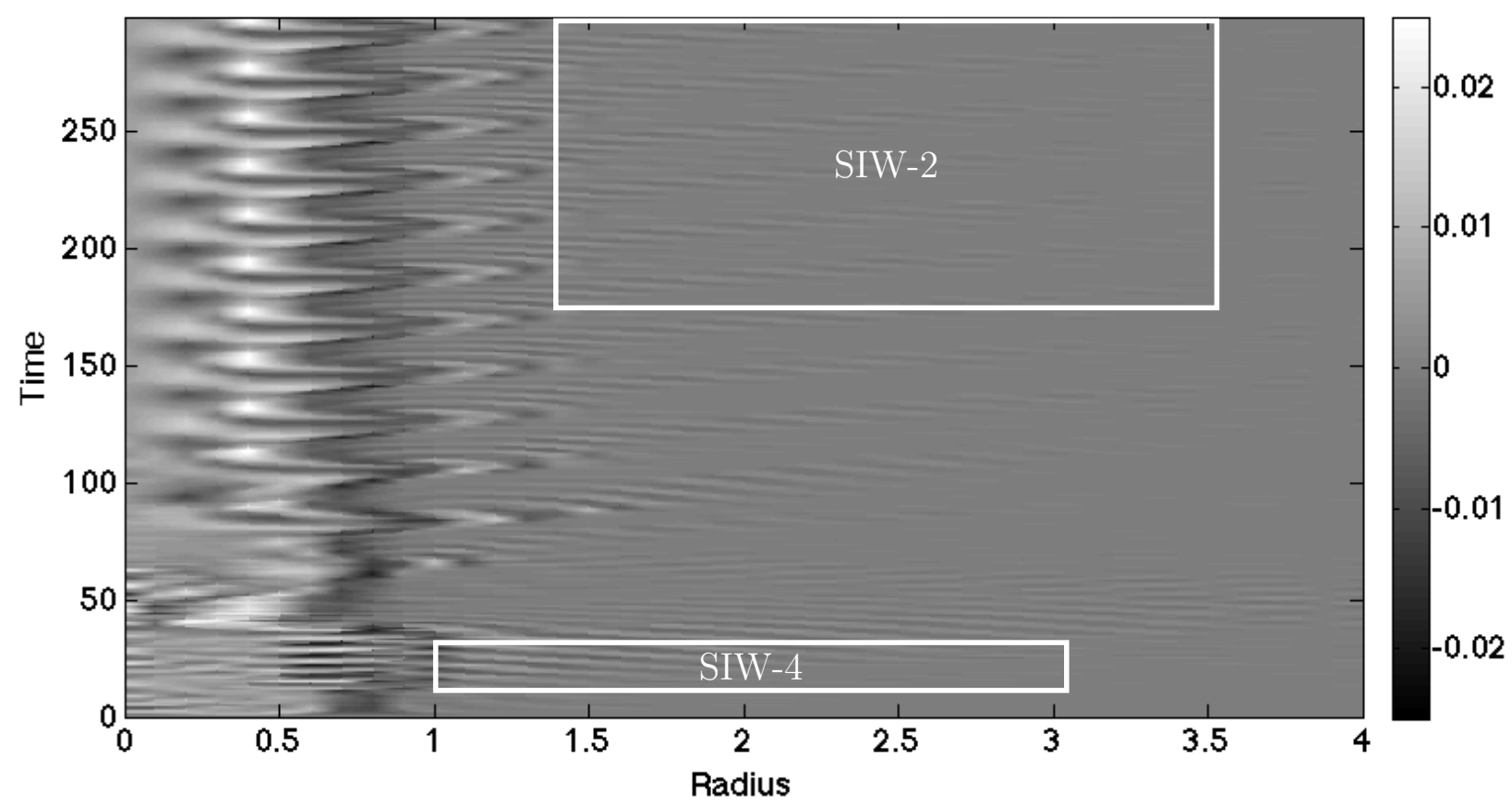

(a)

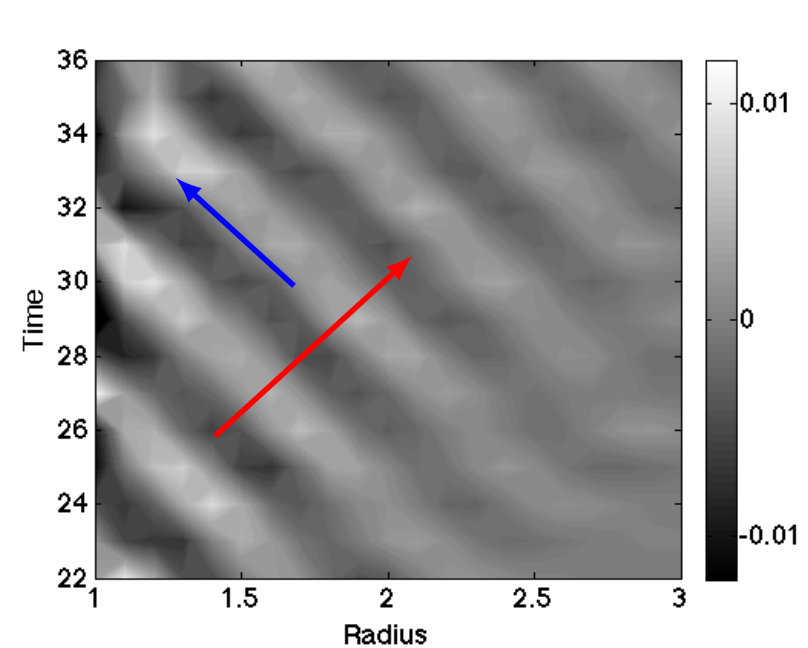

(b)

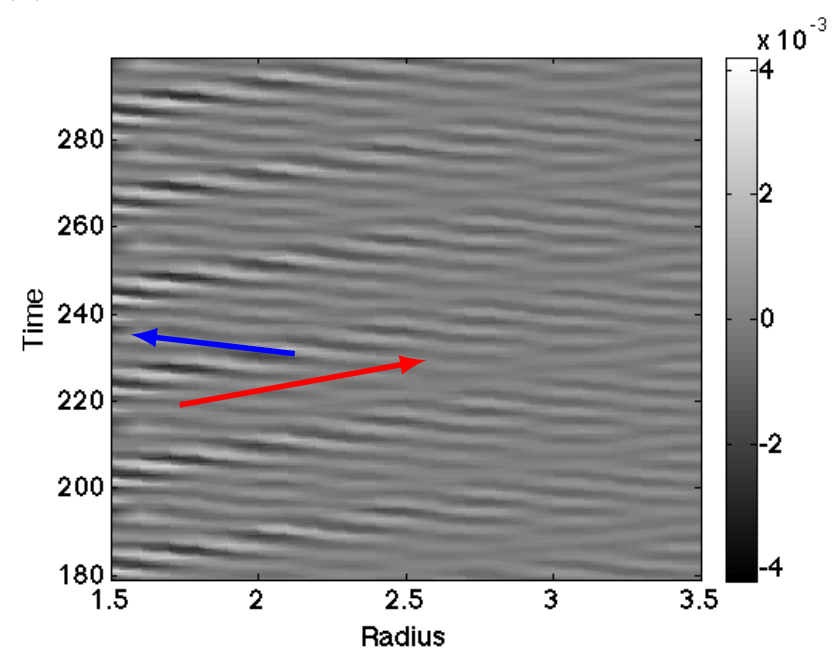

(c)

Figure 6: (a) Hovmöller diagram of vertical velocity on mid-depth horizontal plane, shown in the radius-time section. (b) and (c) show the zoom-in views of regions denoted by the boxes in (a), for phases SIW-4 and SIW-2, respectively. The blue arrows show the direction of phase speed and the red arrows point the direction of group velocity. 


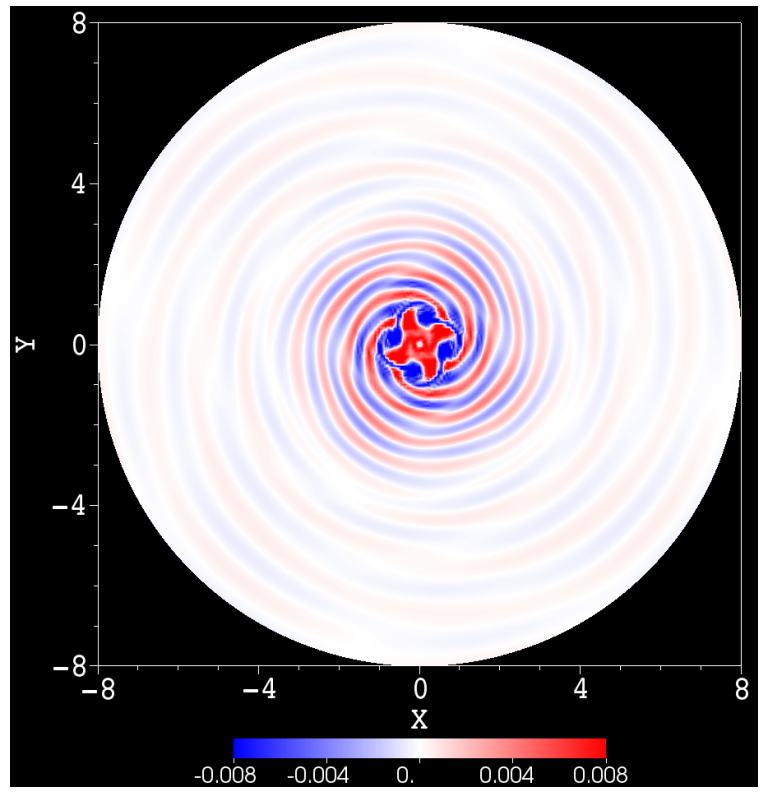

(a)

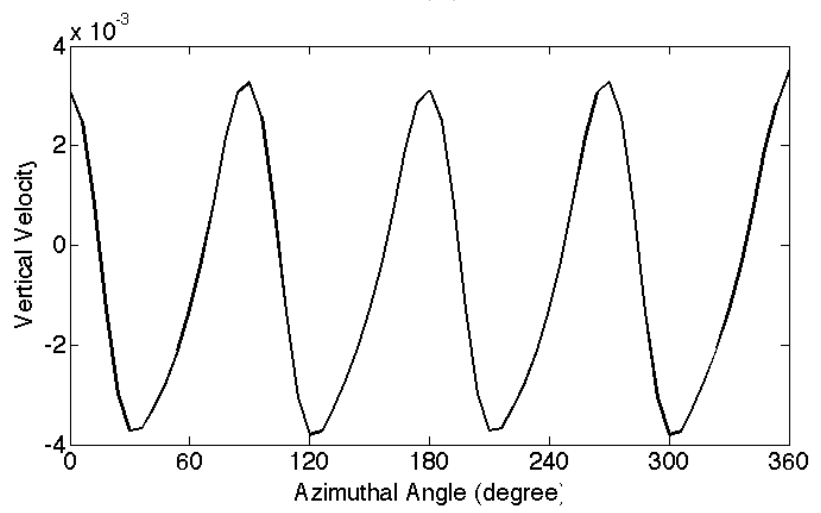

(c)

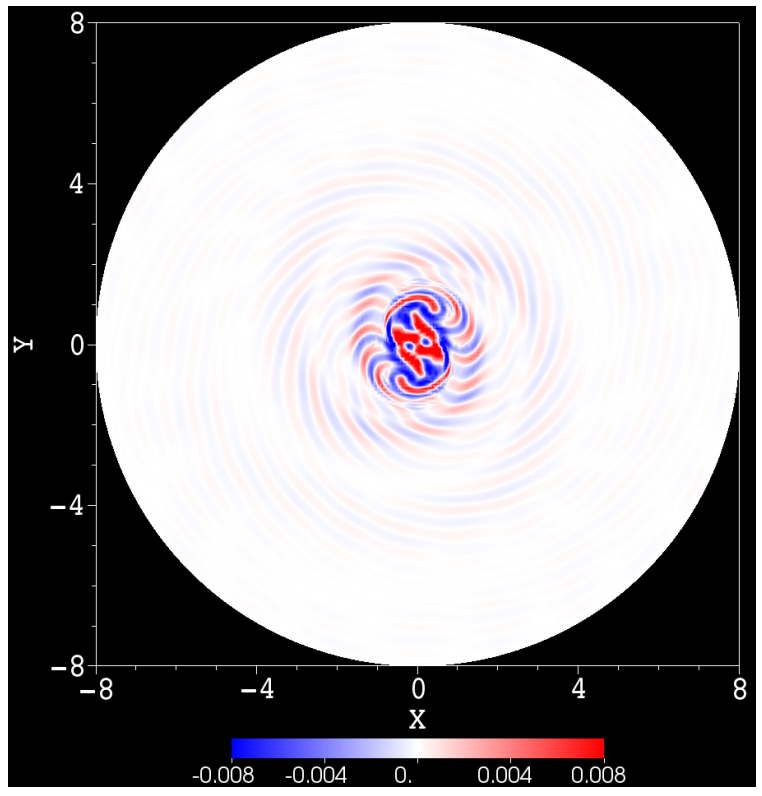

(b)

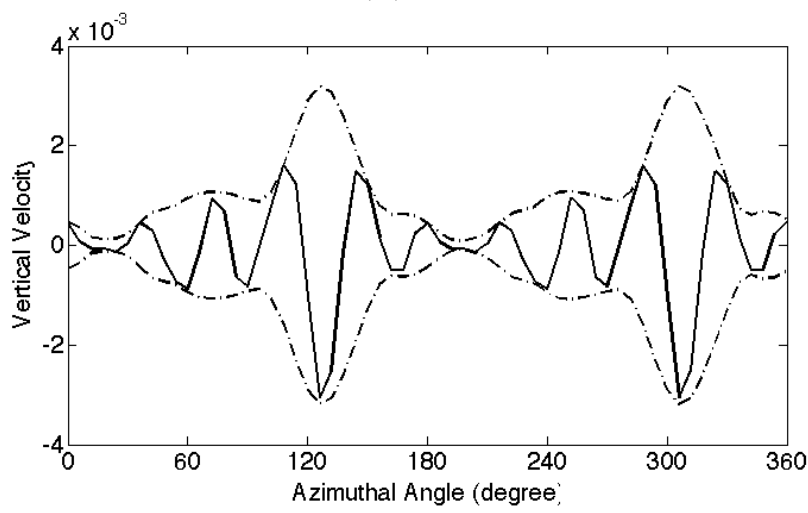

(d)

Figure 7: Upper panel: vertical velocity fields at the mid-depth horizontal plane, from Exp. T9, at (a) time $=30$ and $(\mathrm{b})$ time $=204$. Lower panel: the corresponding vertical velocity along the circle of radius $r=6$ for phase (c) SIW-4 and (d) SIW-2 (dashed lines outlining the wave envelope). 


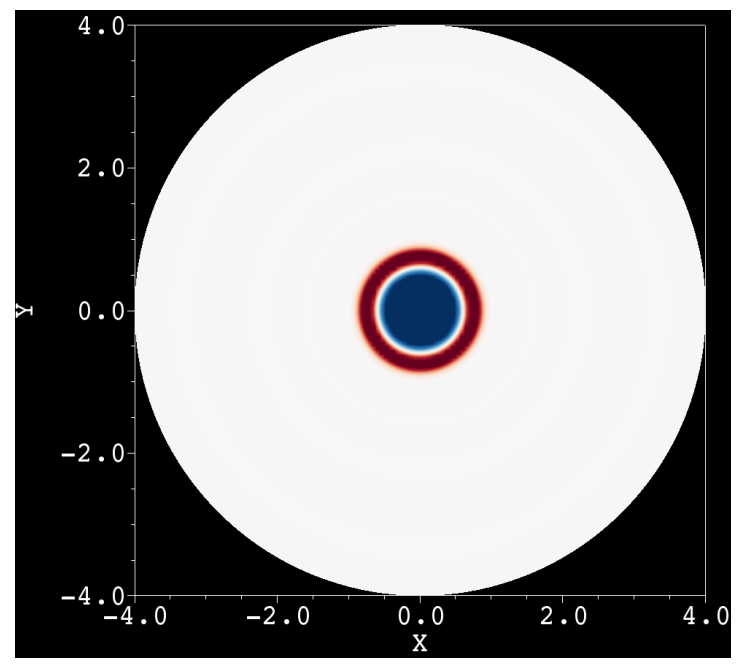

(a)

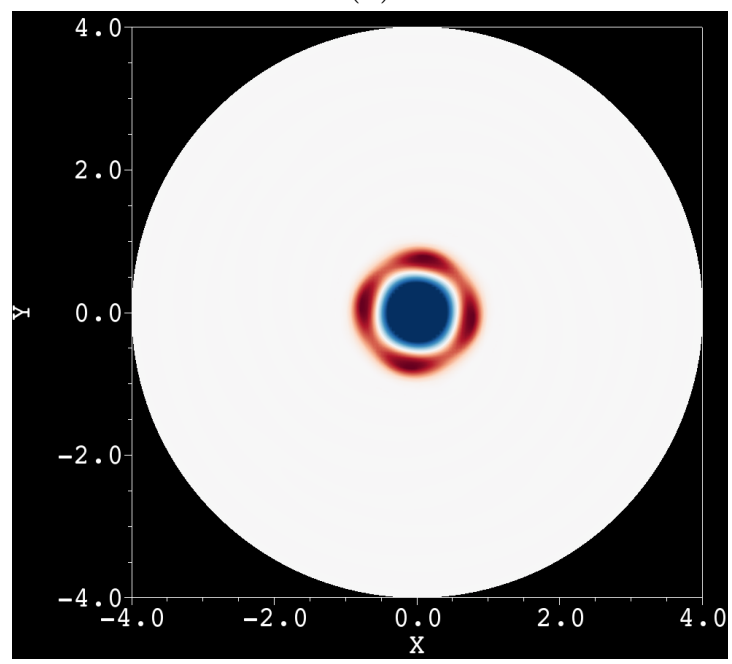

(c)

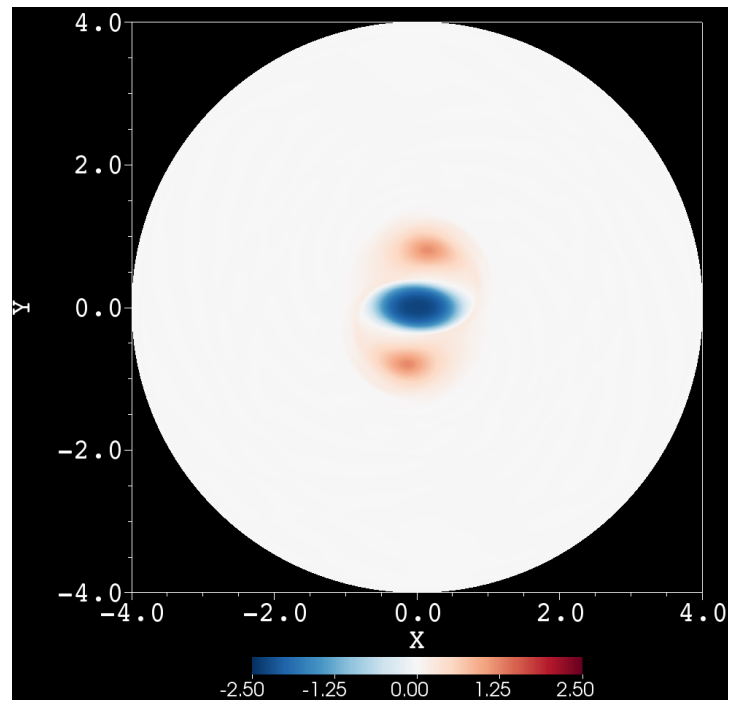

(e)

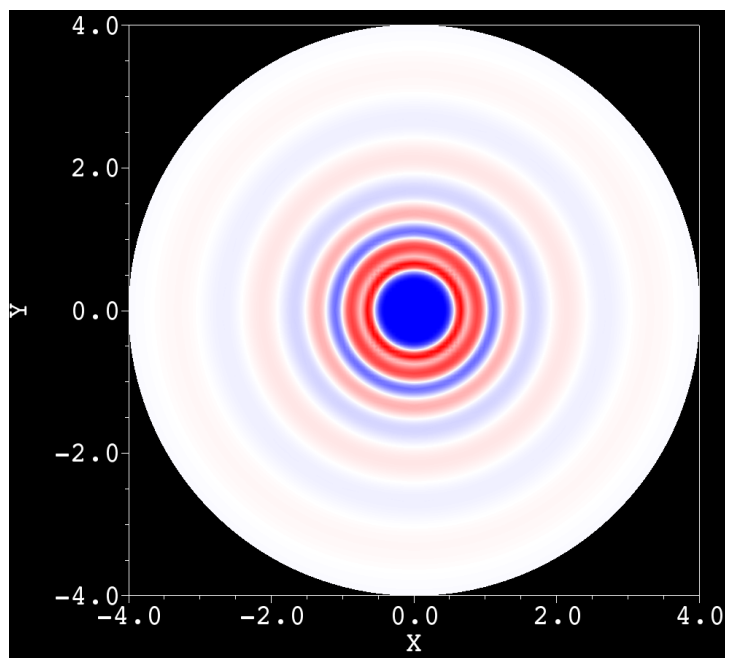

(b)

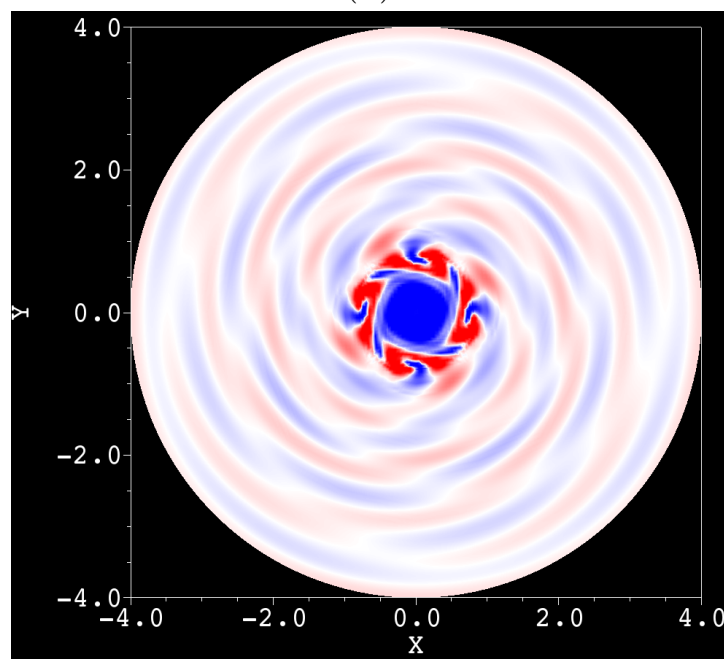

(d)

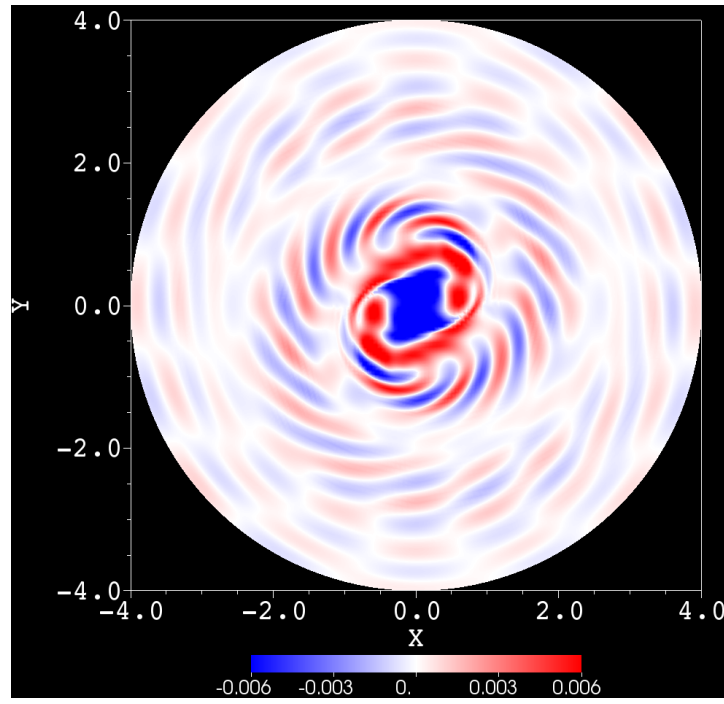

(f)

Figure 8: Vertical vorticity fields (left column) and vertical velocity fields (right column) from Exp. T10; both fields are plotted at mid-depth horizontal plane at $(\mathrm{a}, \mathrm{b})$ time $=6 ;(\mathrm{c}$, d) time $=26$ and $(\mathrm{e}, \mathrm{f})$ time $=205$. 


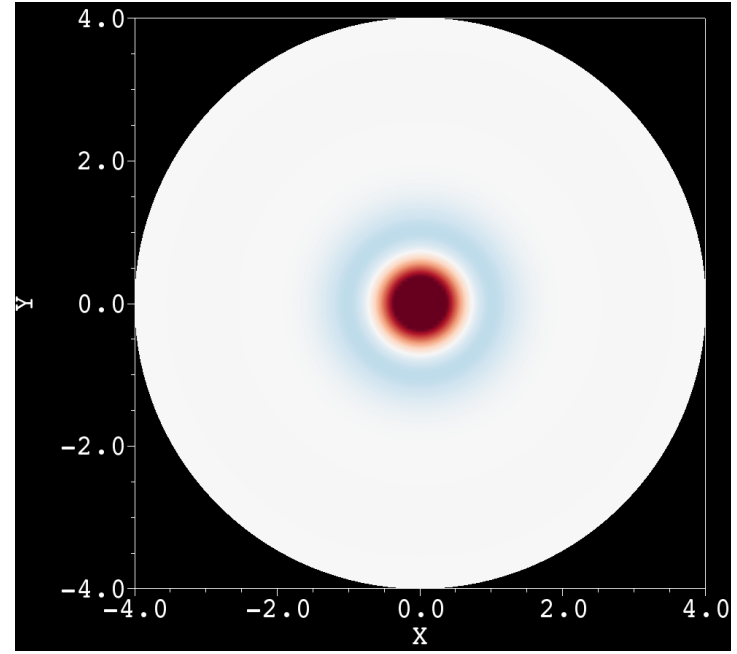

(a)

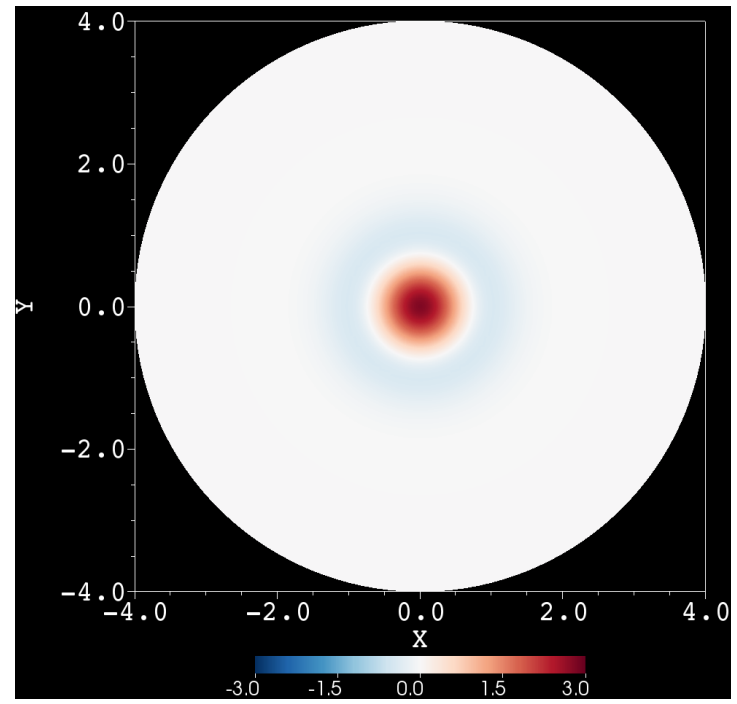

(c)

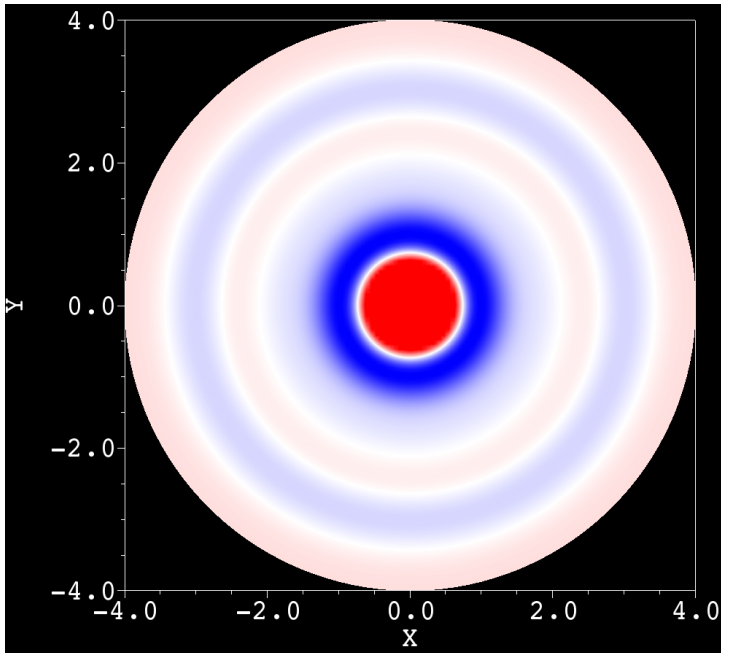

(b)

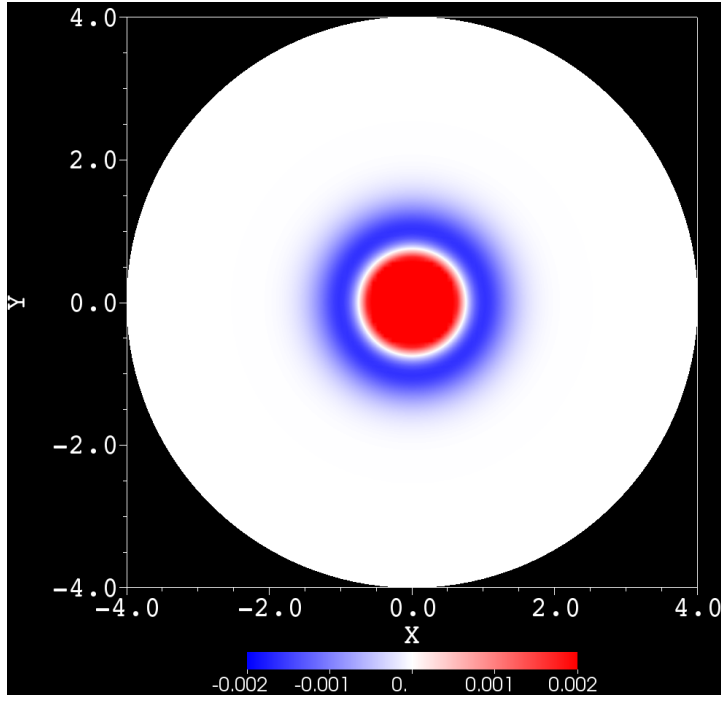

(d)

Figure 9: Vertical vorticity fields (left column) and vertical velocity fields (right column) from Exp. C1; both fields are plotted at mid-depth horizontal plane at (a,b) time $=10$ and $(\mathrm{c}, \mathrm{d})$ time $=296$. 

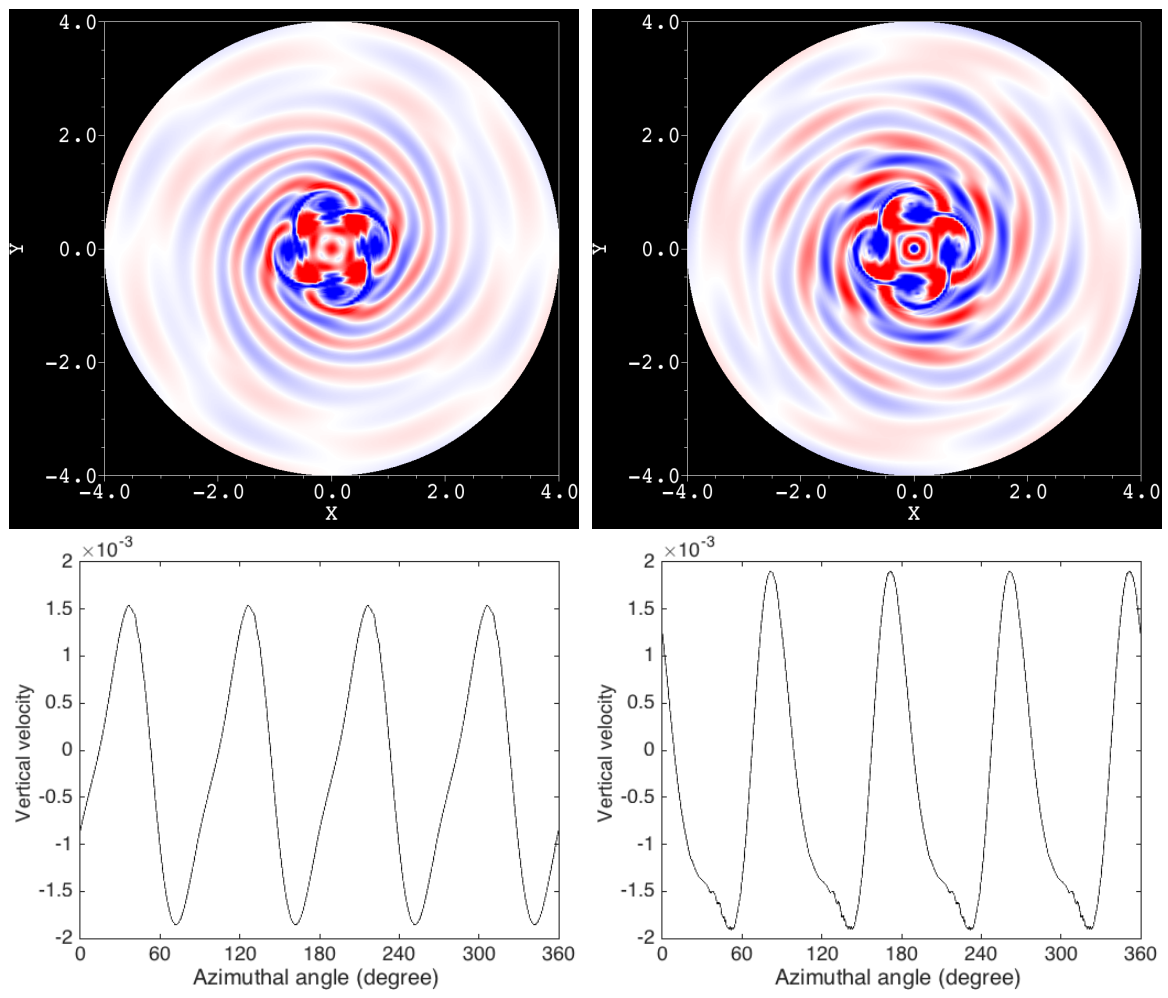

(a)

(b)
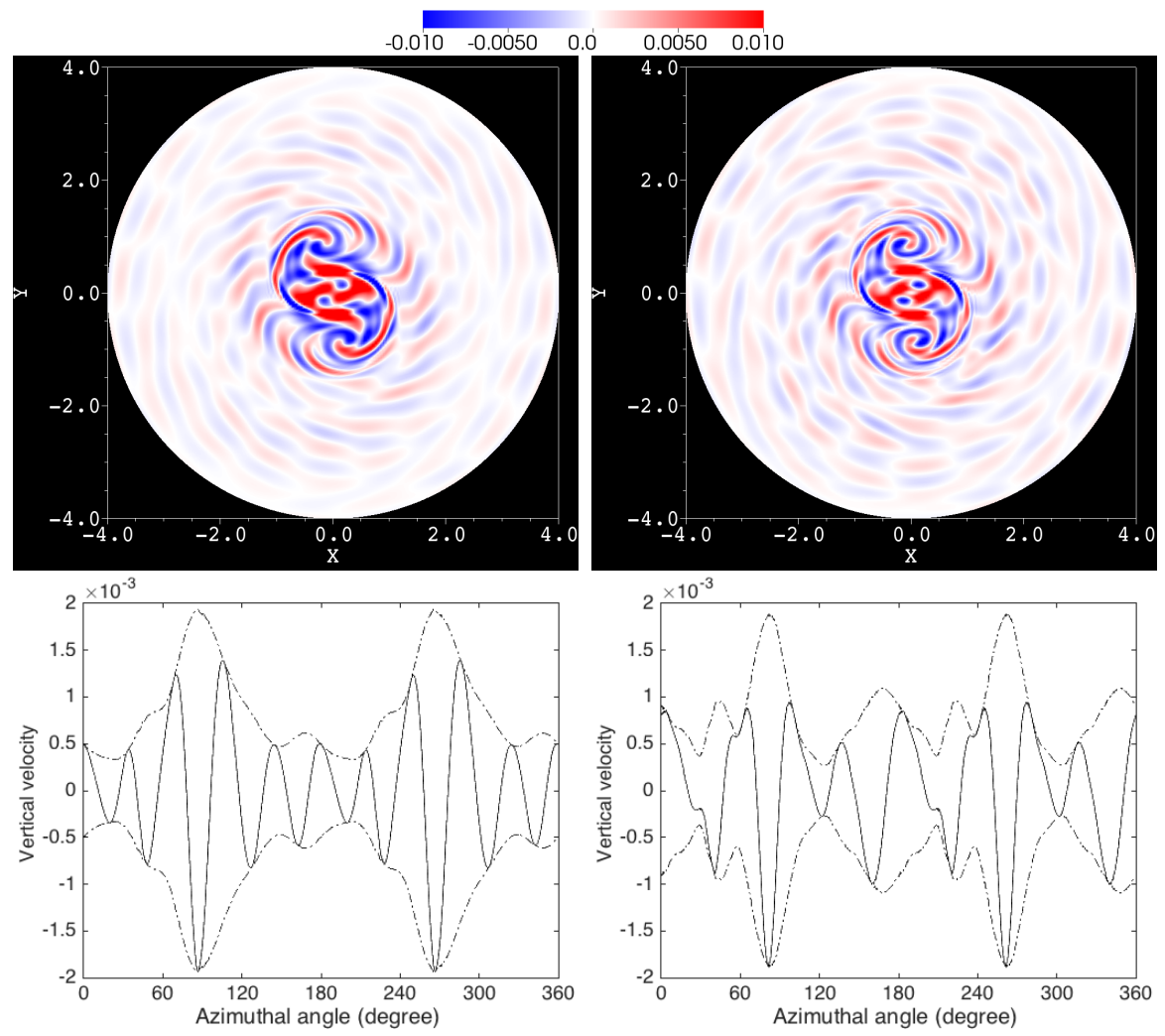

(c)

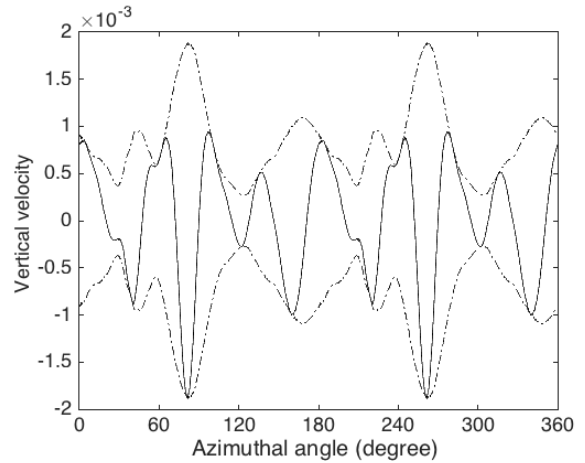

(d)

Figure 10: Phases SIW-4 and SIW-2 of (a,c) Exp. T11 and (b,d) Exp. T12 at the mid-depth horizontal plane. [The vertical velocities are sampled along the circle of radius $=2.5$ to show the four complete waves and two wave packets; dashed lines represent wave envelopes.] 


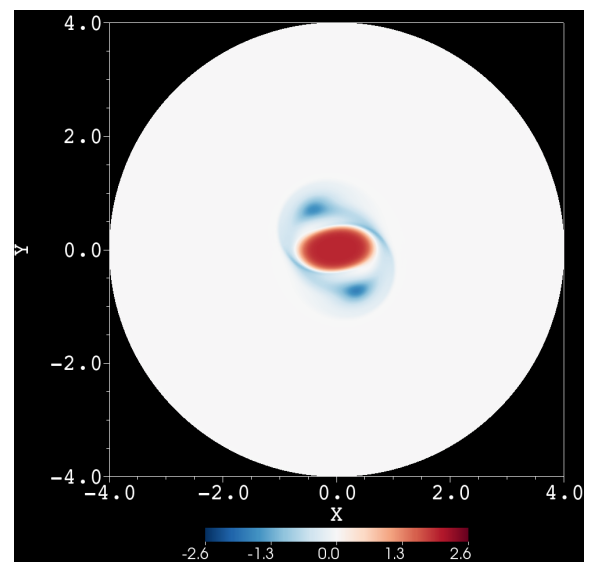

(a)

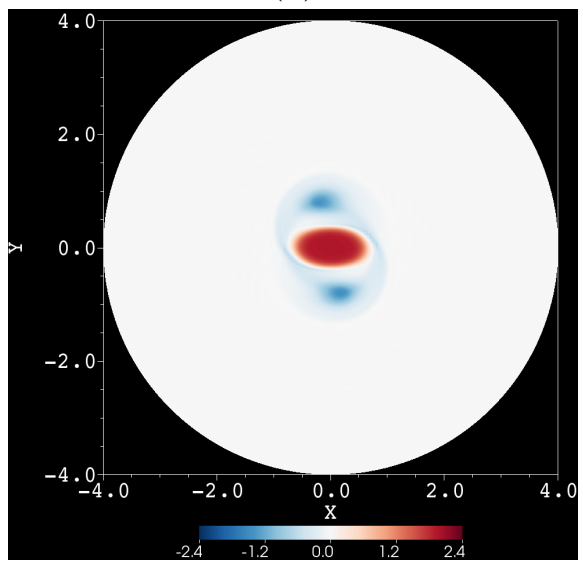

(c)

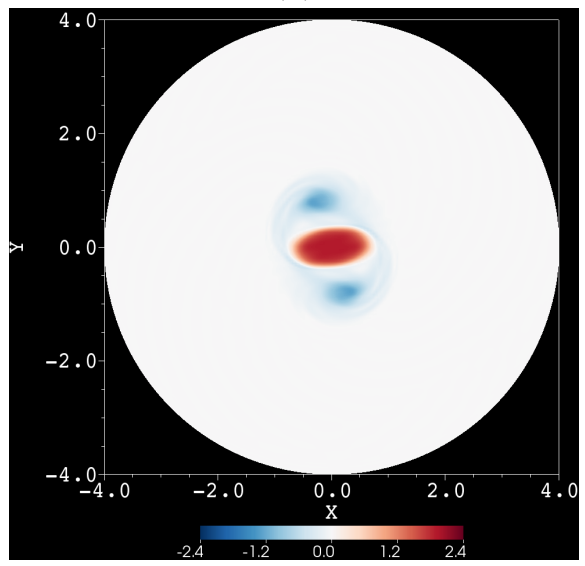

(e)

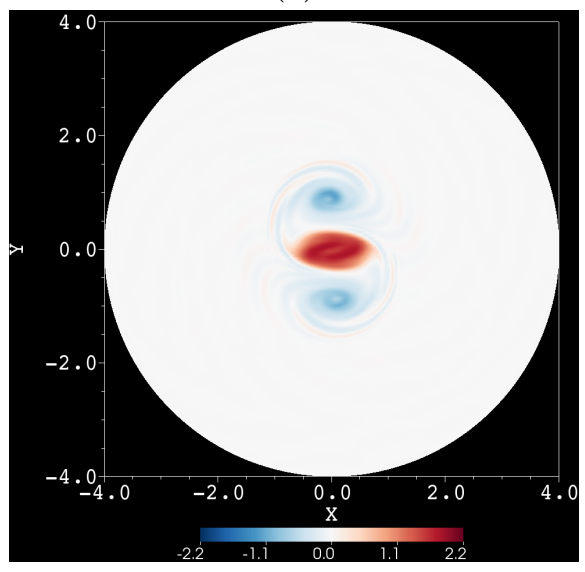

(g)

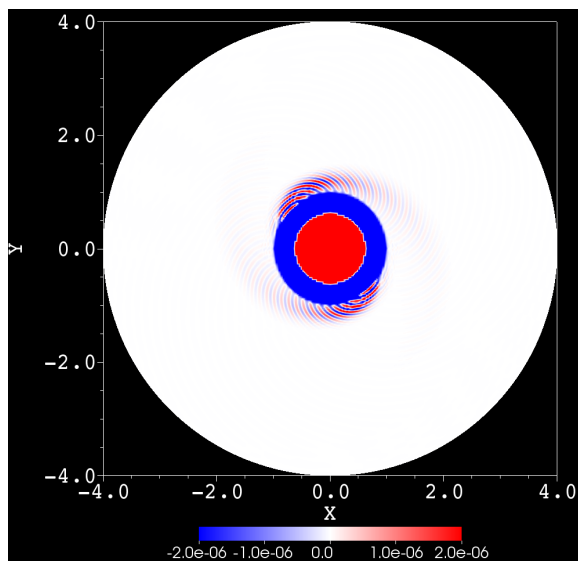

(b)

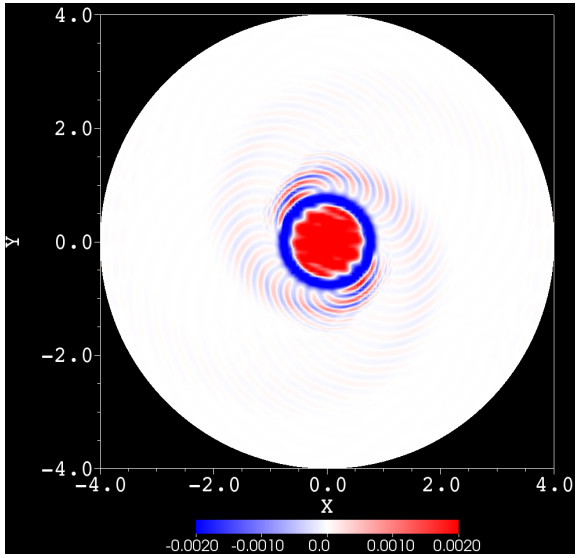

(d)

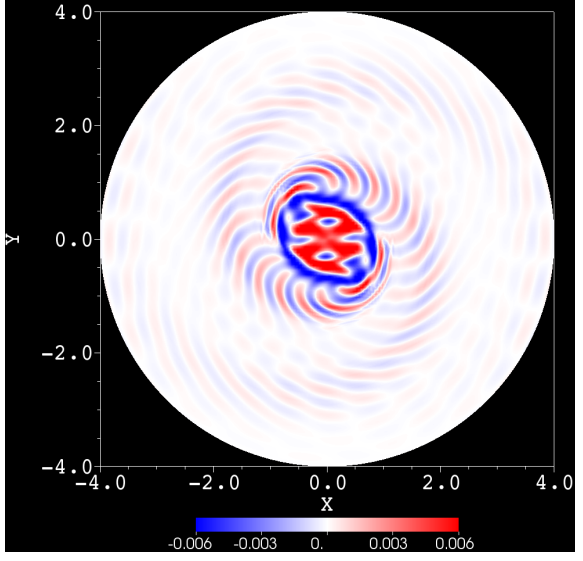

(f)

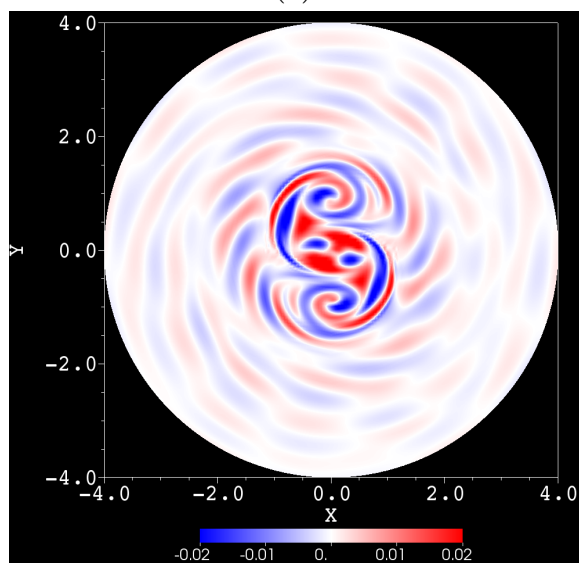

(h) 


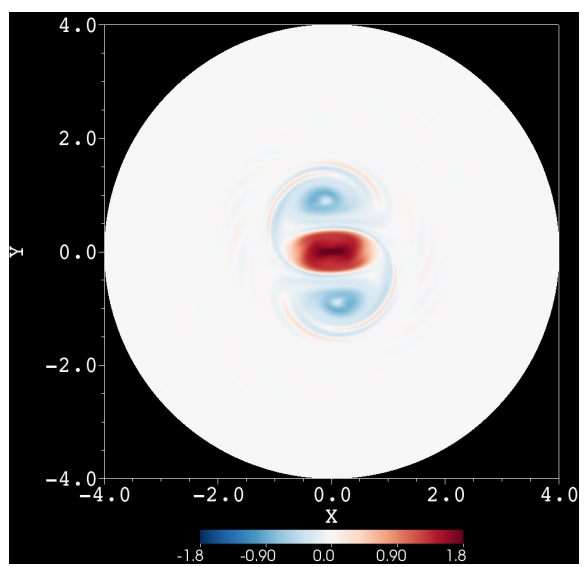

(i)

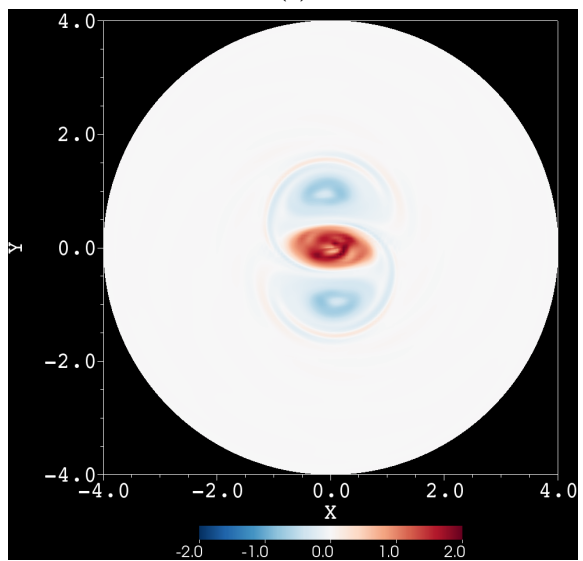

$(\mathrm{k})$

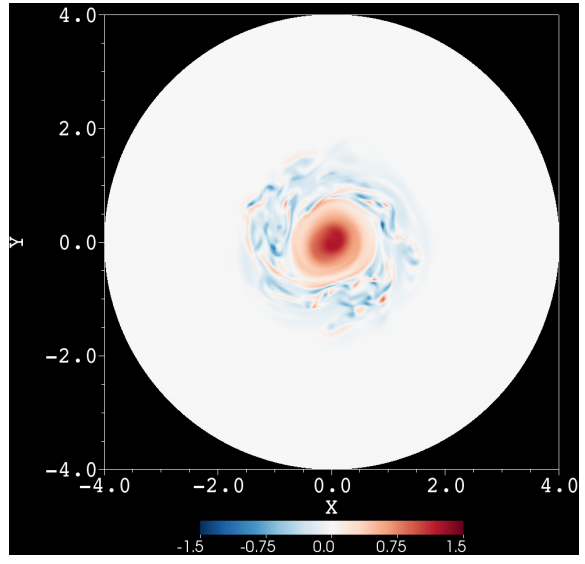

$(\mathrm{m})$

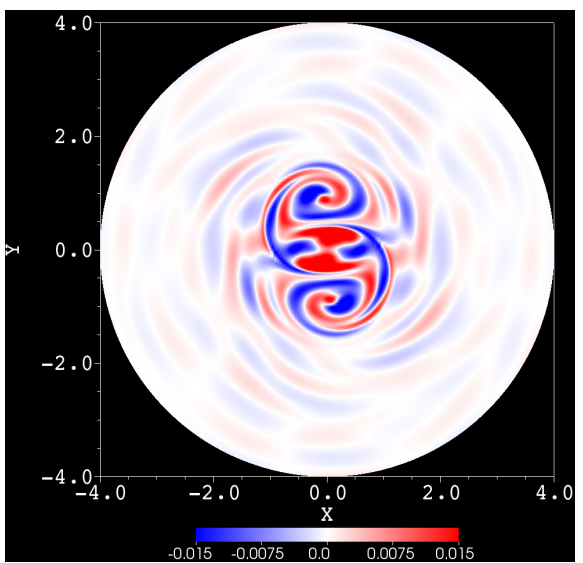

(j)

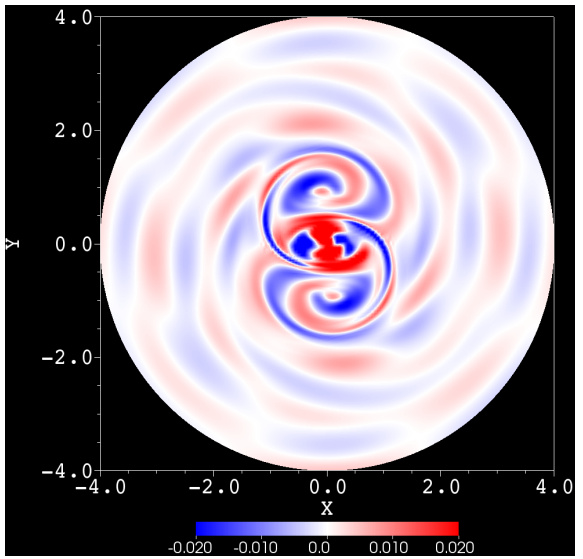

(l)

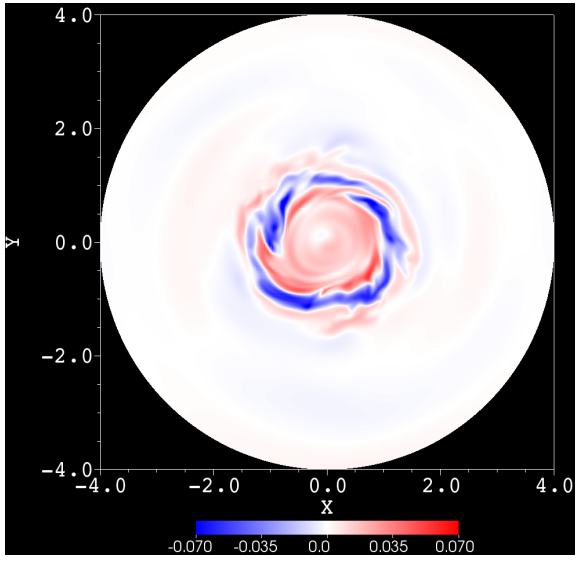

$(\mathrm{n})$

Figure 11: Snapshots of vertical vorticity fields (left column) and the corresponding vertical velocity fields (right column) at mid-depth horizontal plane for target experiments (a,b) T2; (c,d) T3; (e,f) T4; (g,h) T5; (i,j) T6; (k,l) T7; (m,n) T8. (Note the change of magnitude of vertical velocity.) 


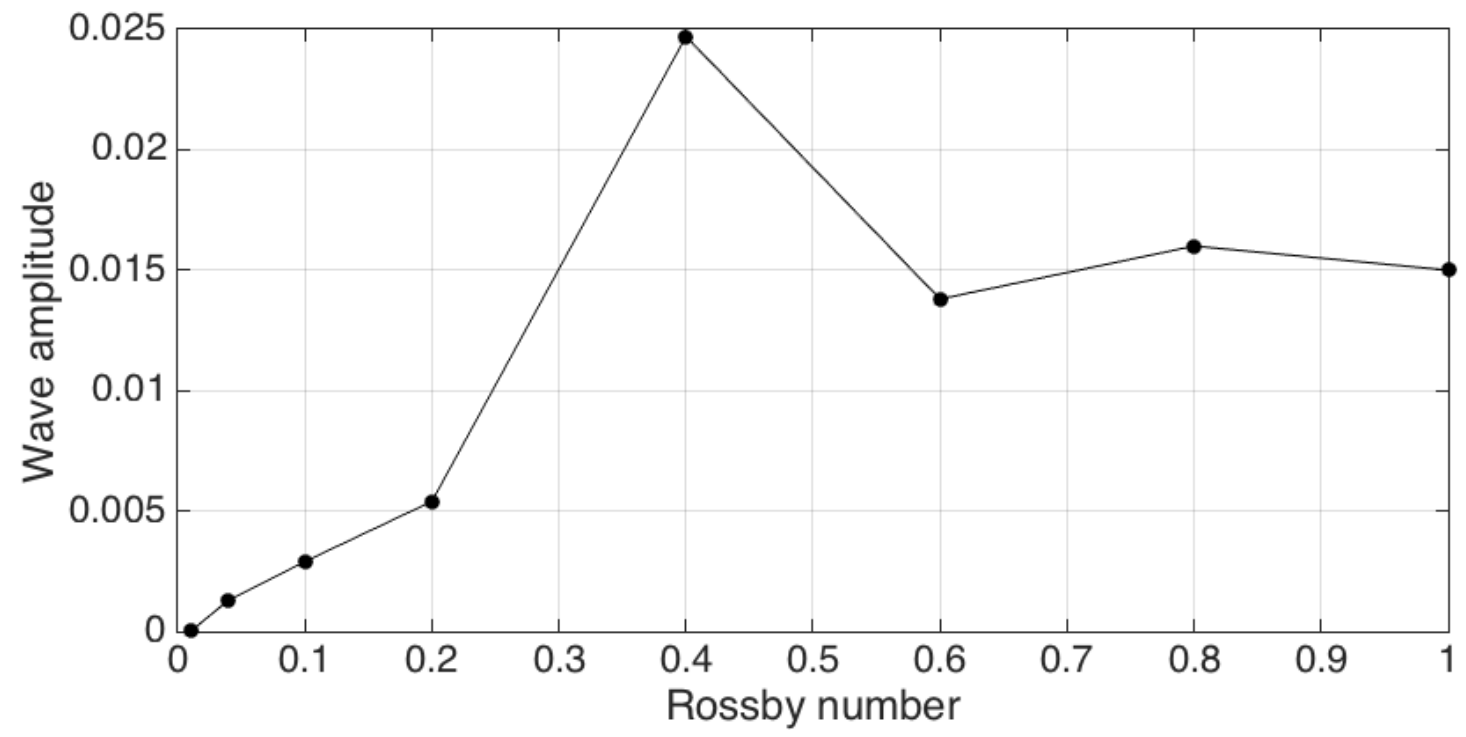

(a)

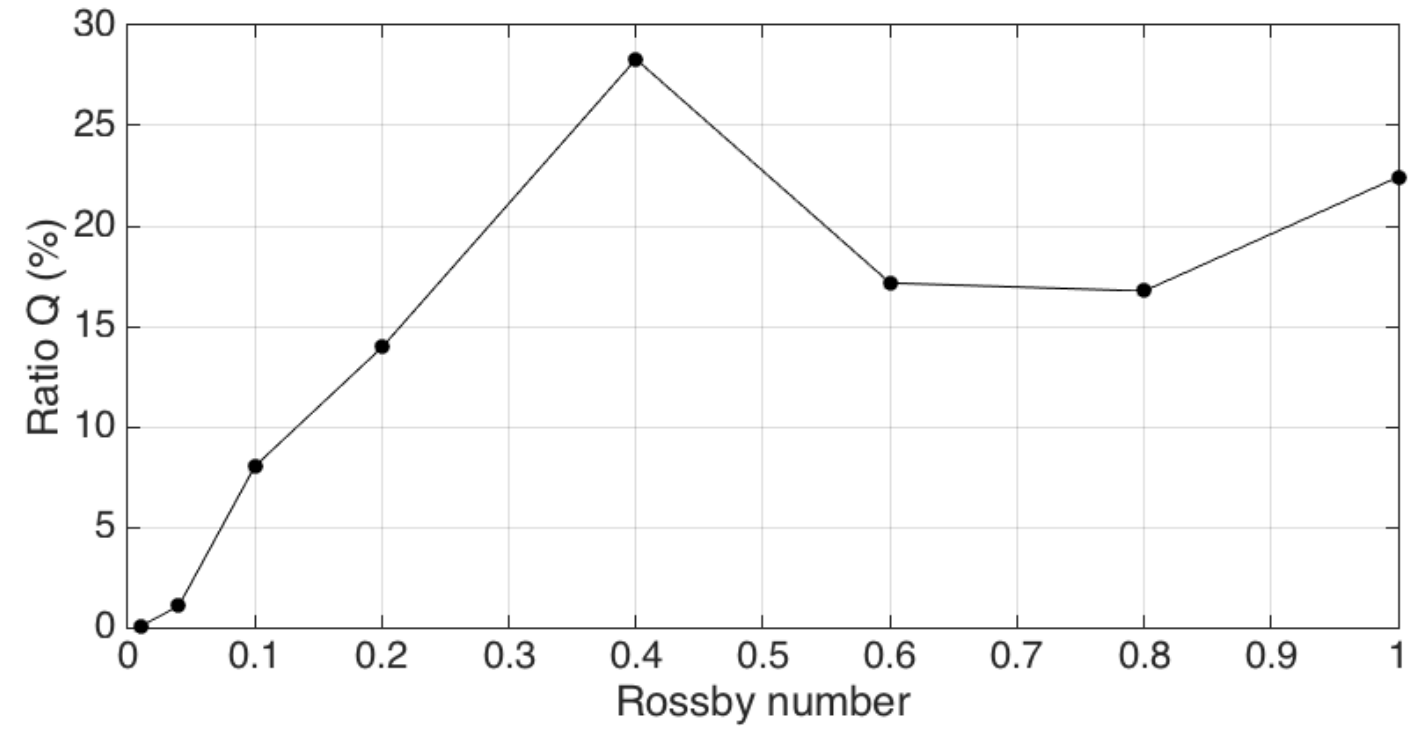

(b)

Figure 12: The change of (a) SIWs amplitude and (b) ratio $Q$ with Rossby number. (Wave amplitudes are recorded at the final stage of vortex evolution.) 


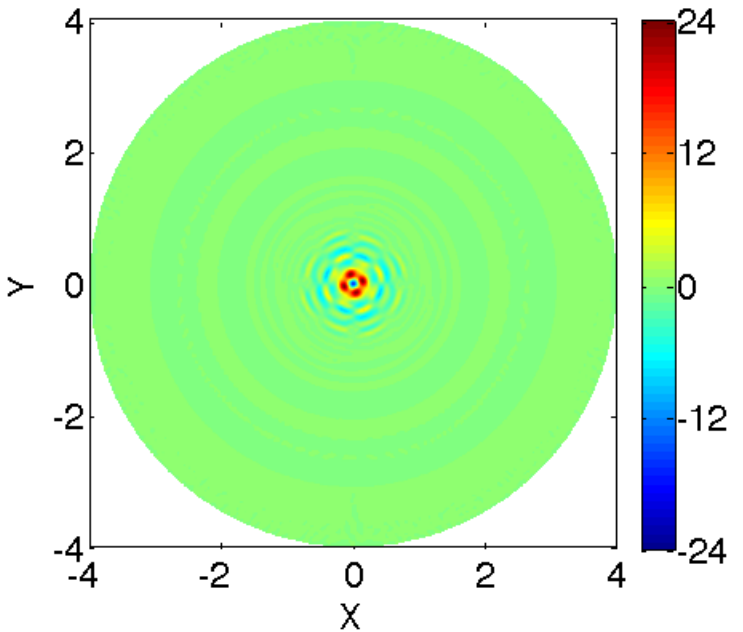

(a)

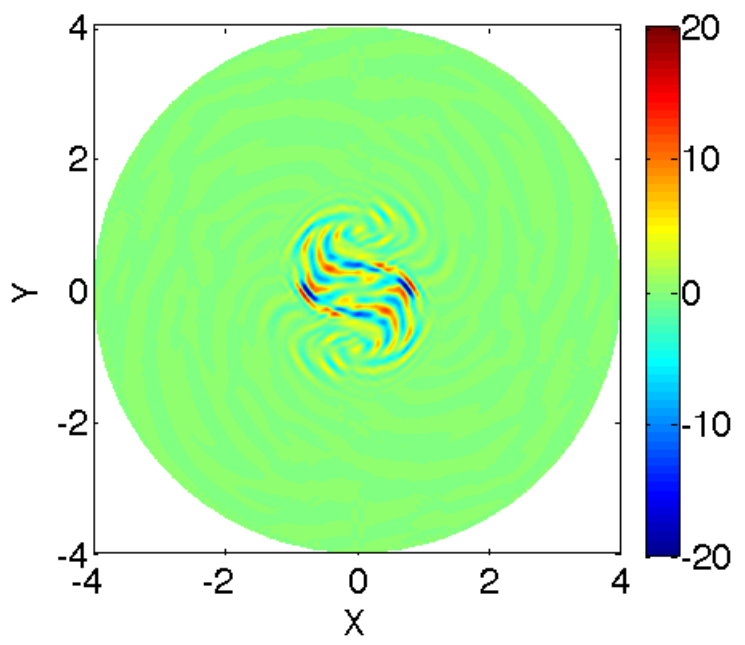

(c)

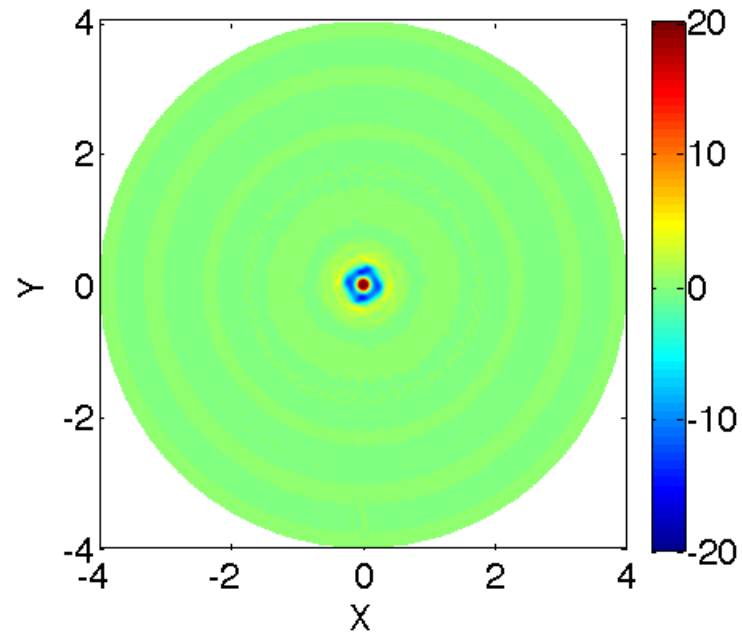

(b)

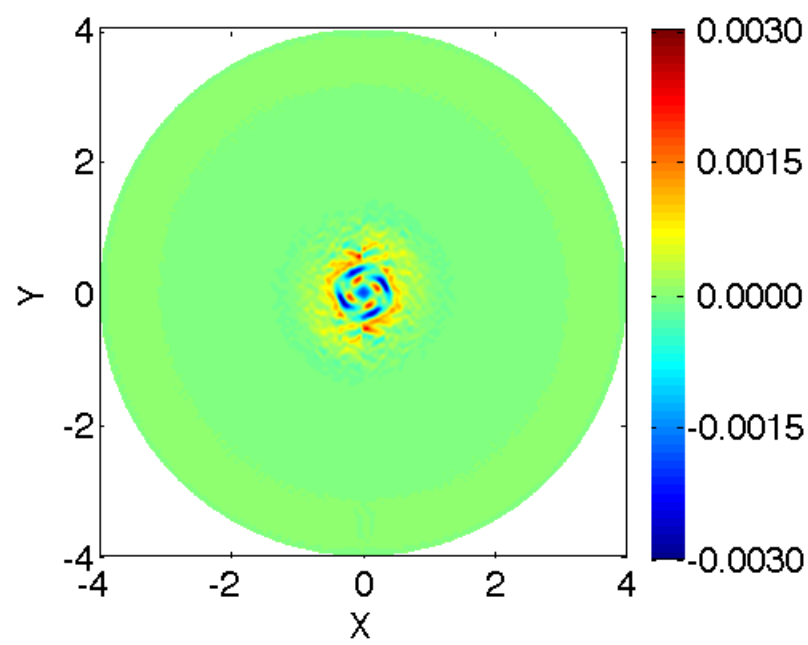

(d)

Figure 13: Wave source fields $S$ in Exp. T1 (left column) at (a) time $=6$, (c) time $=204$; the fields in Exp. C1 (right column) at (b) time $=10,(\mathrm{~d})$ time $=296$, at mid-depth horizontal plane. (Note the change of magnitude of wave source.) 


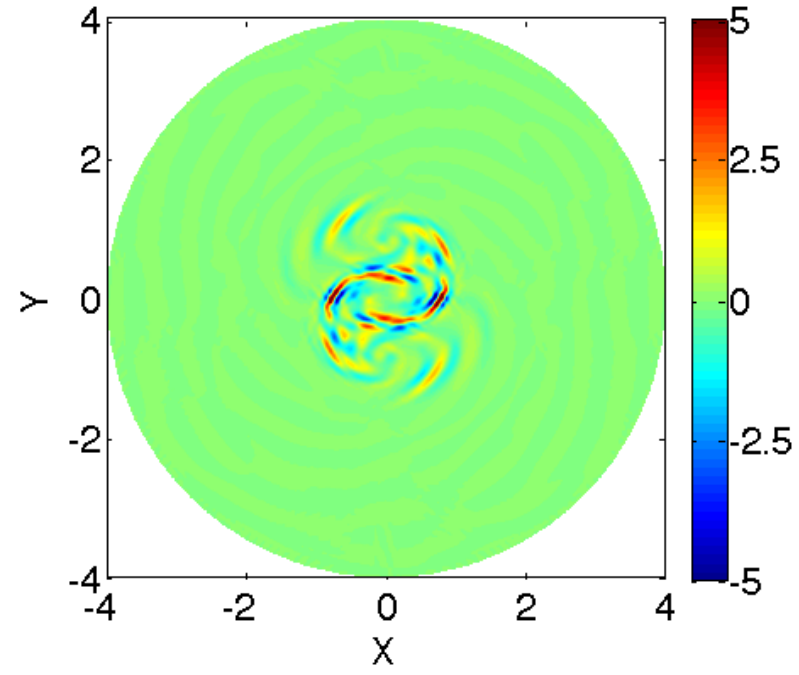

(a)

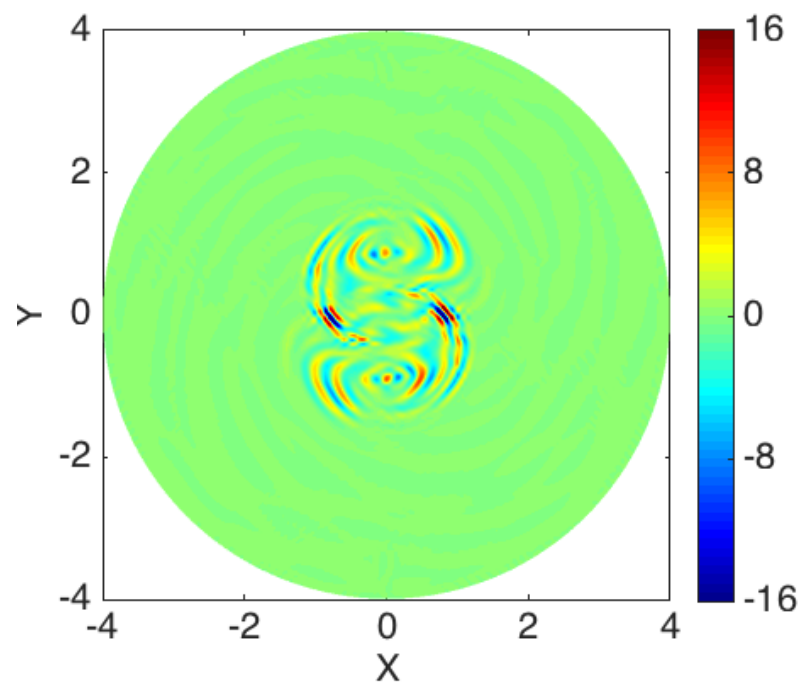

(c)

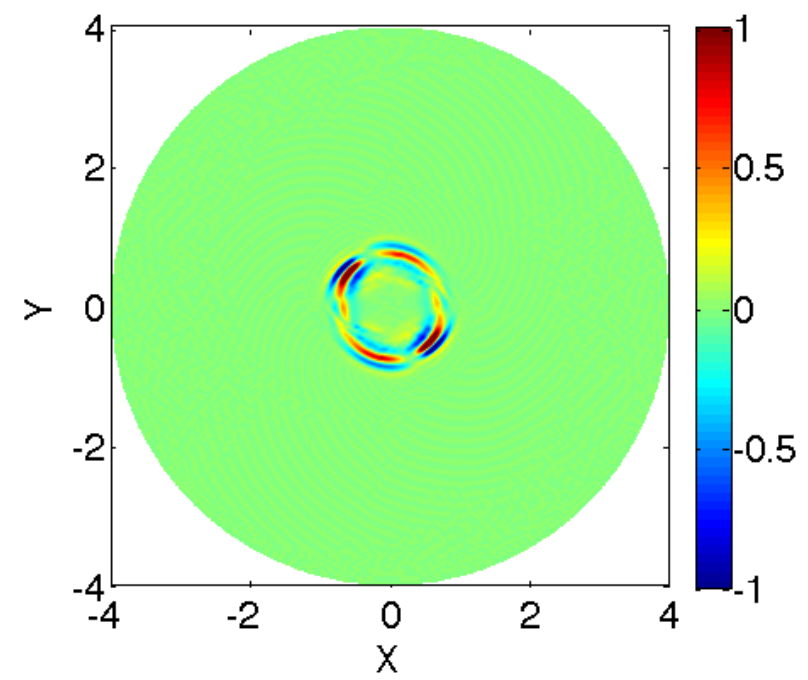

(b)

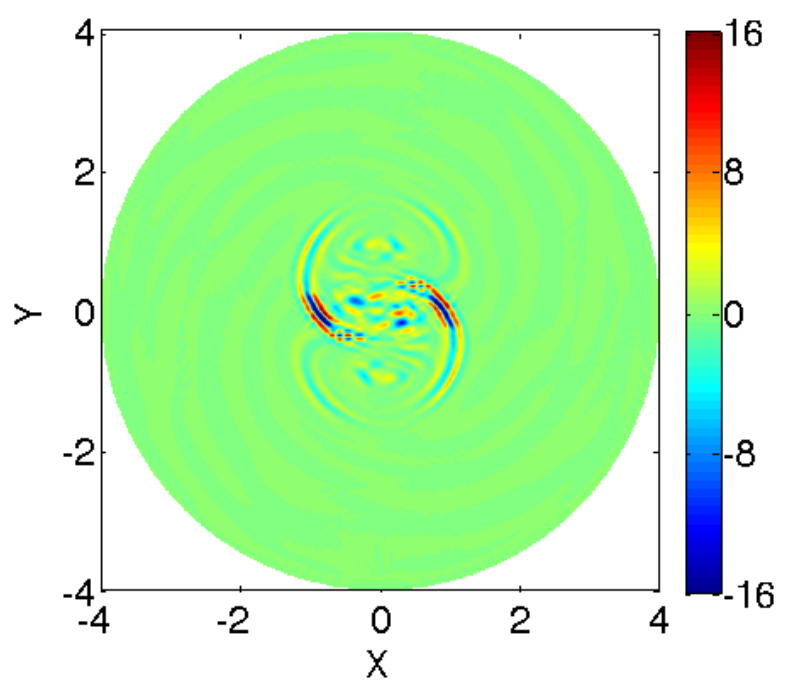

(d)

Figure 14: Snapshots of the wave source fields $S$ in Exps. (a) T10 (b) T2 (c) T5 and (d) T7 at mid-depth horizontal plane. (The time stamps are the same to that of the corresponding wave fields shown in Figs. 8f, 11b, 11h and 11l, respectively.) 


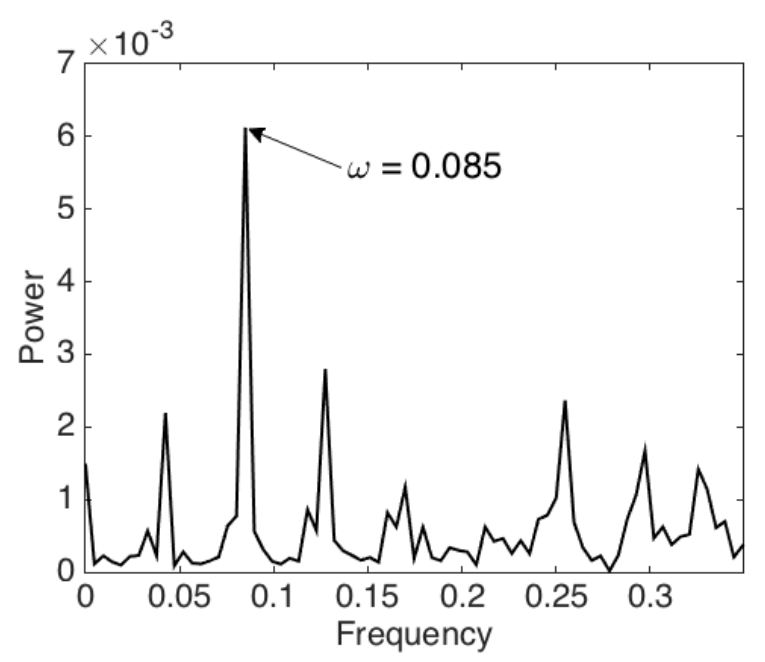

(a)

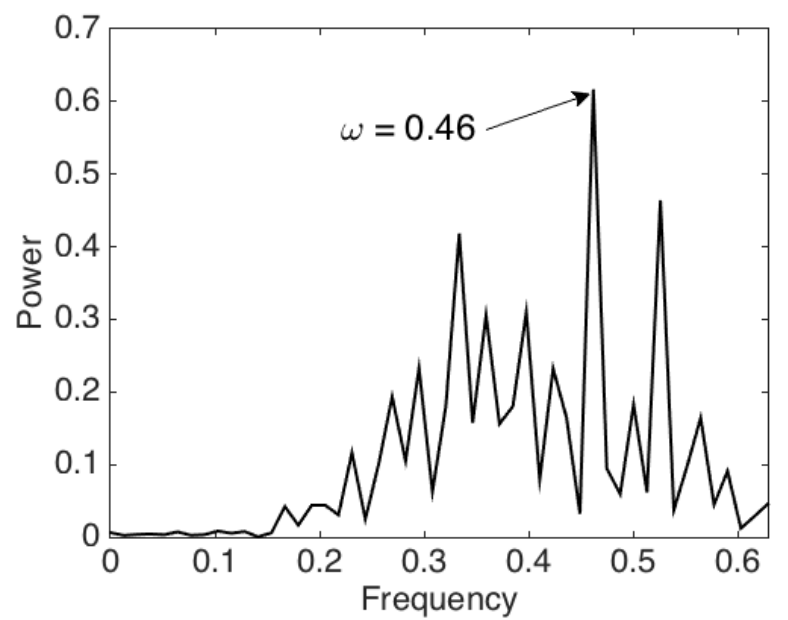

(c)

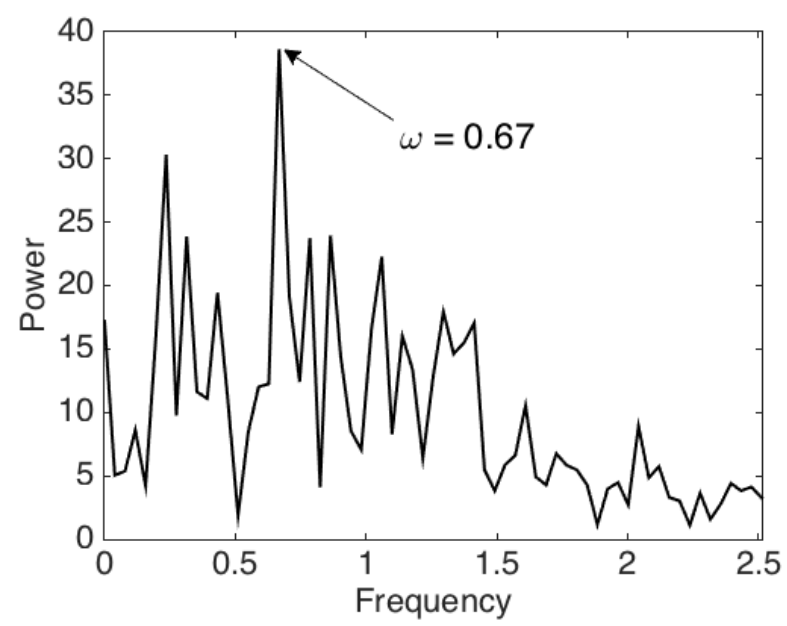

(e)

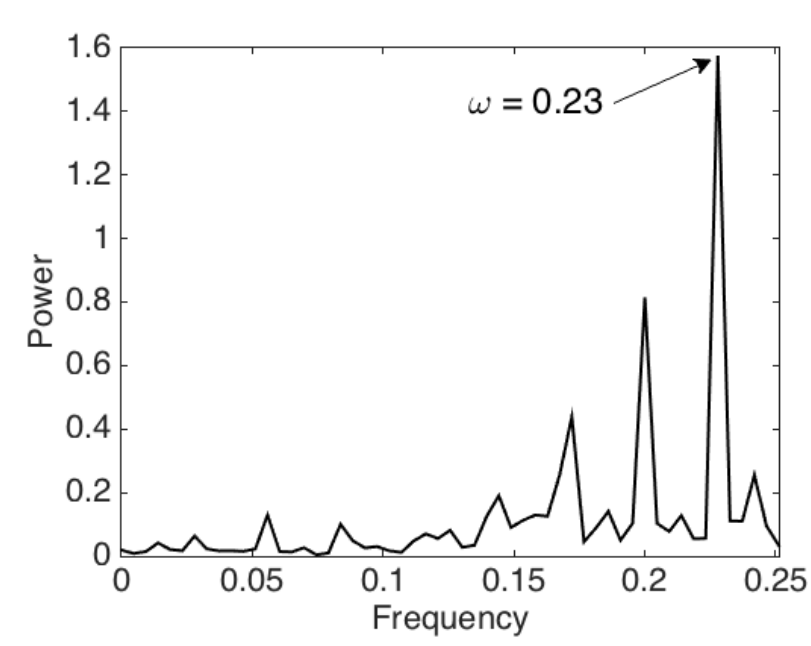

(b)

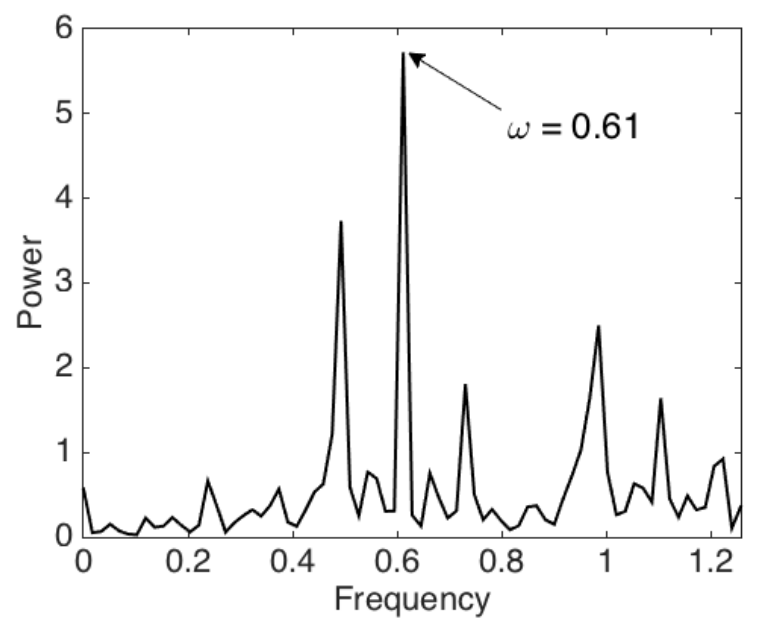

(d)

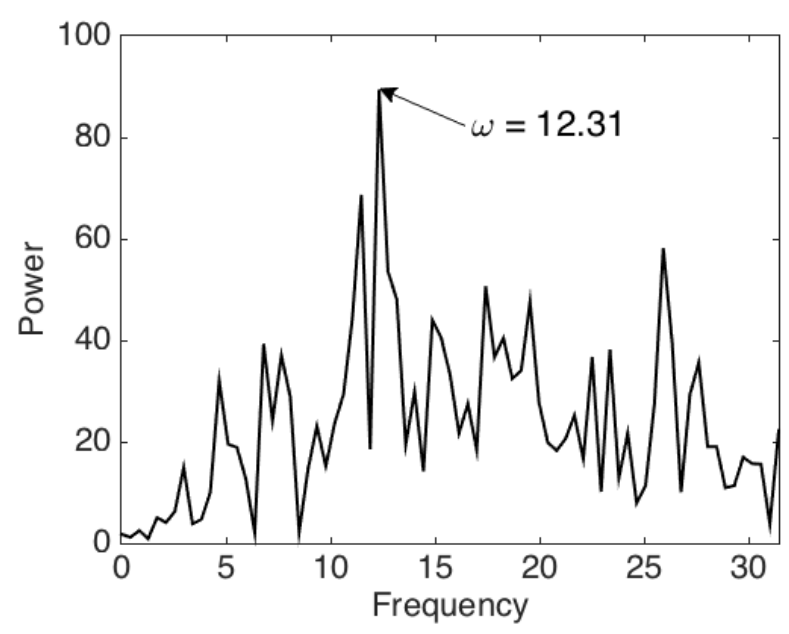

(f)

Figure 15: Frequency spectra of the wave source $S$ inside the vortex in Exps. (a) T2 (b) T3 (c) T4 (d) T1 (e) T5 and (f) T8. (Note that the maximum frequency of inertial waves is 2 according to Eq. 4.) 


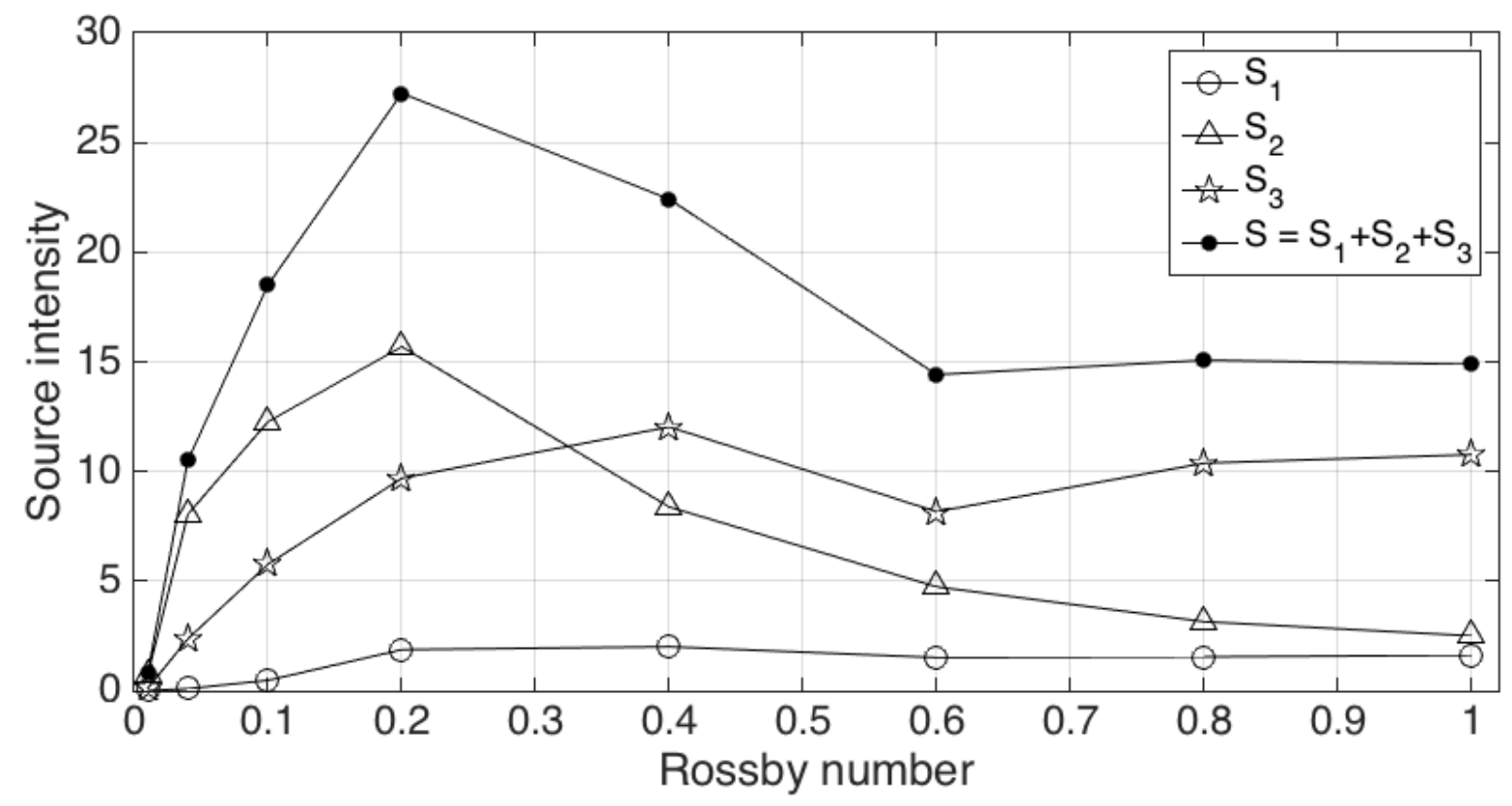

Figure 16: The change of source intensity with Rossby number. (Source intensities are computed at the final stage of vortex evolution.) 


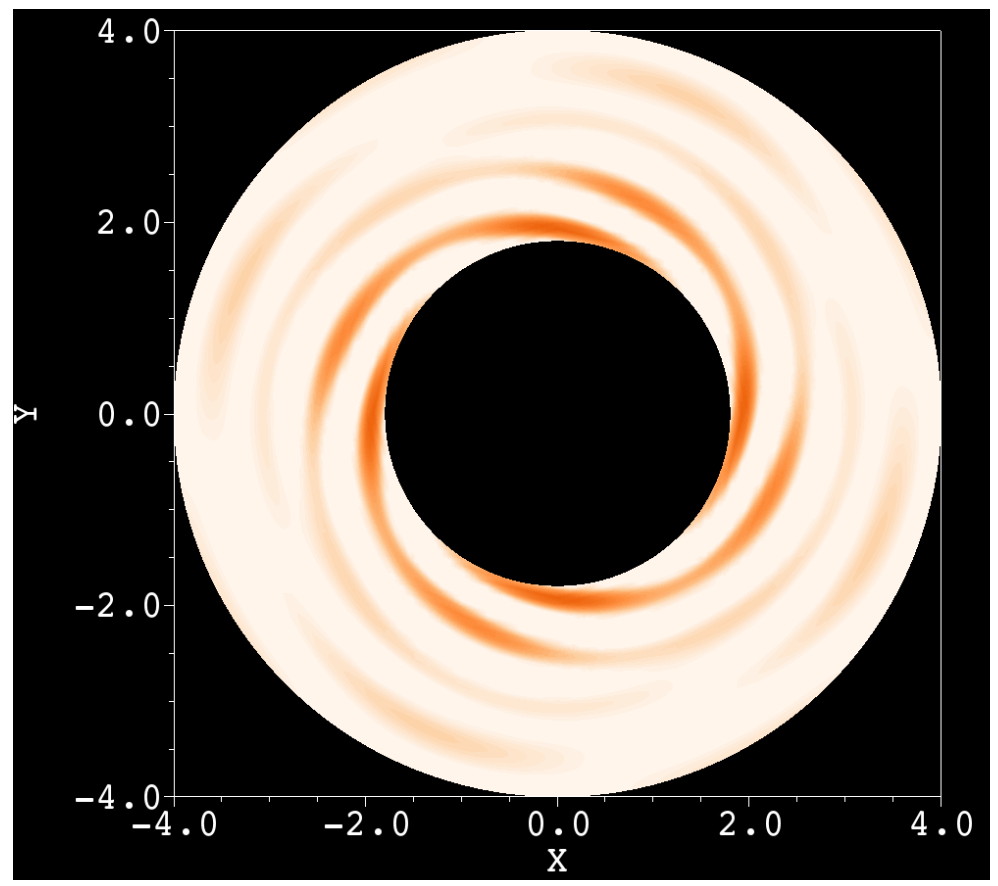

(a)

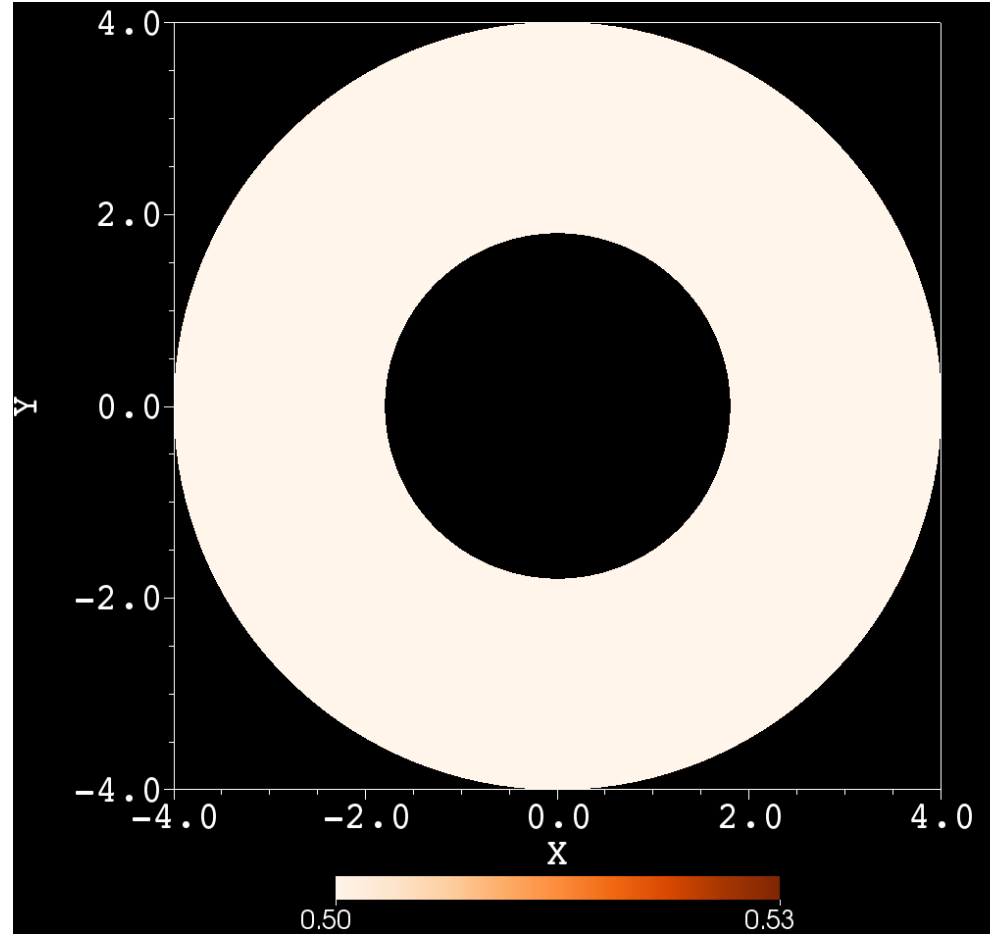

(b)

Figure 17: Tracer fields at mid-depth horizontal plane in (a) Exp. T1 and (b) Exp. C1 at time $=30$. (The central regions occupied by the vortices are clipped to emphasize the outside wave field.) 


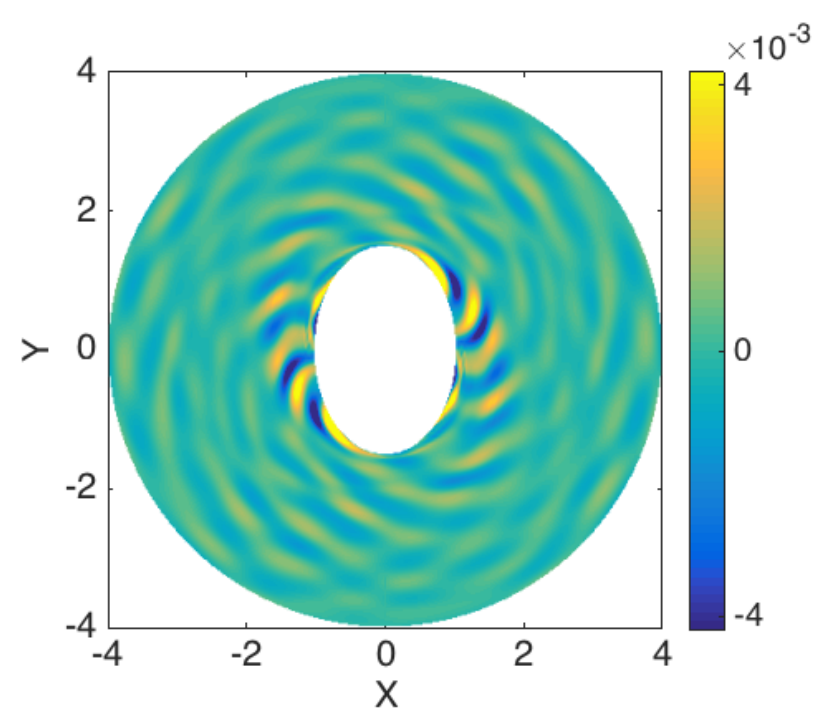

(a)

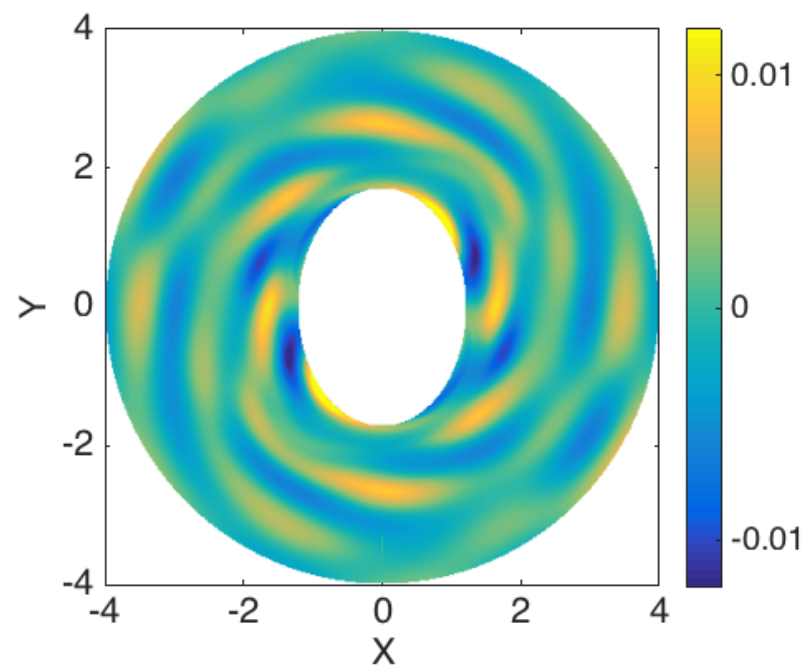

(c)

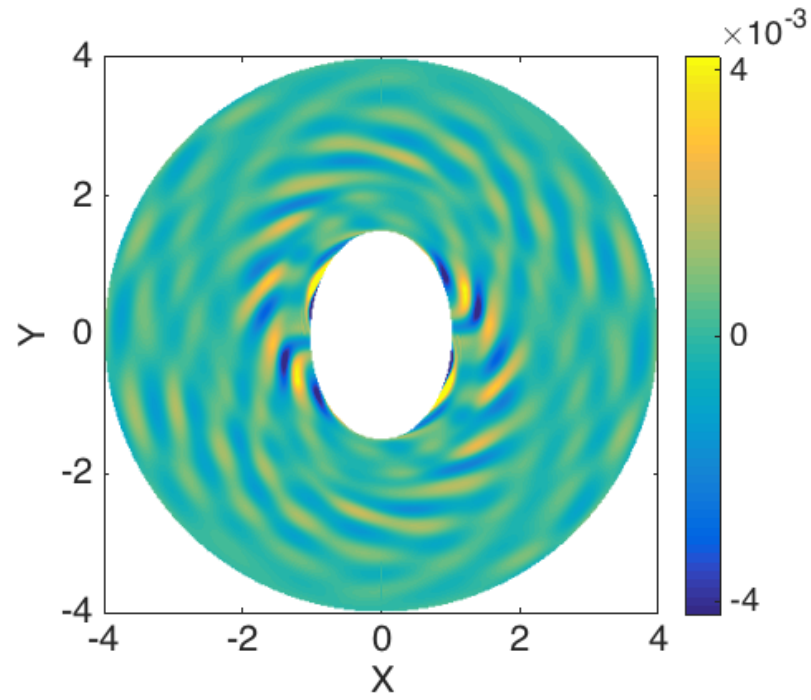

(b)

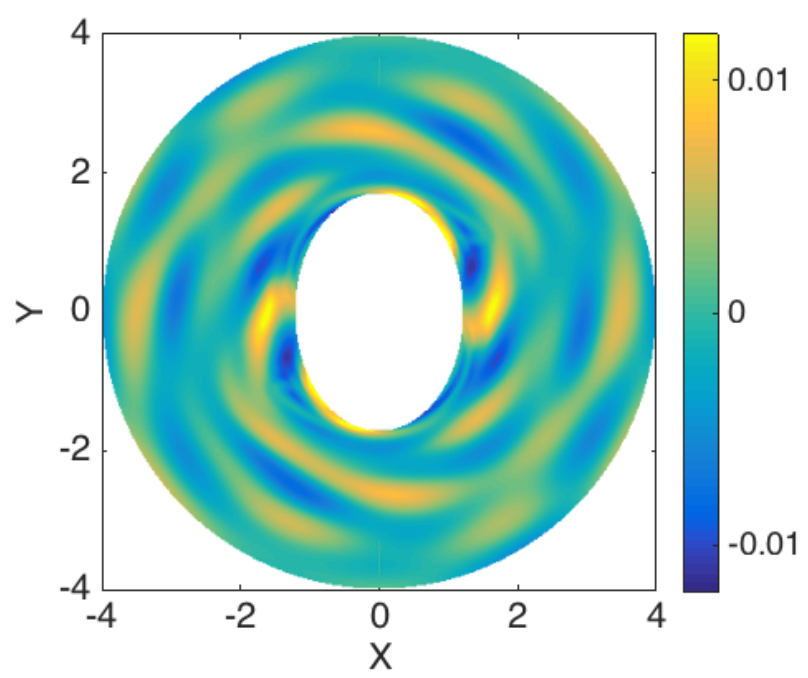

(d)

Figure 18: Snapshots of the vertical tracer advection by SIWs $\left(w \frac{\partial C}{\partial z}\right)$, across mid-depth horizontal plane of (a,b) Exp. T1 and (c,d) Exp. T7; left column: $P e=10^{4}$, right column: $P e=10^{5}$. (The central regions occupied by the vortices are clipped to emphasize the outside wave field.) 


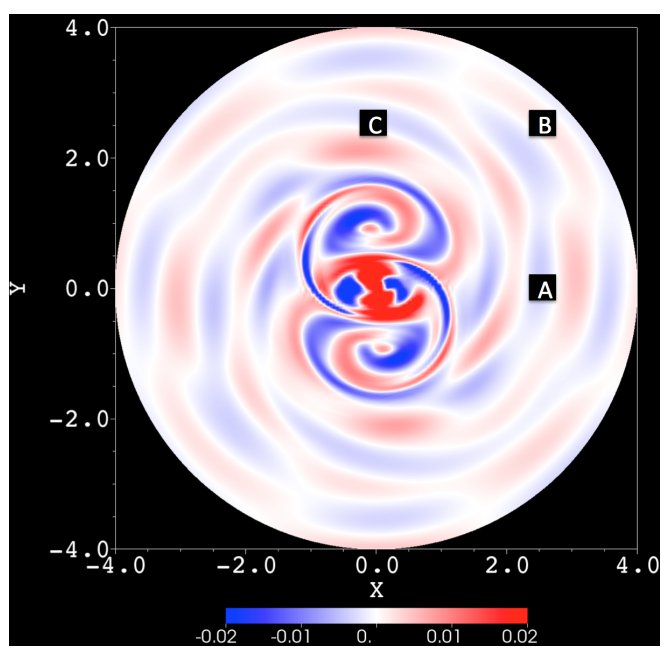

(a)

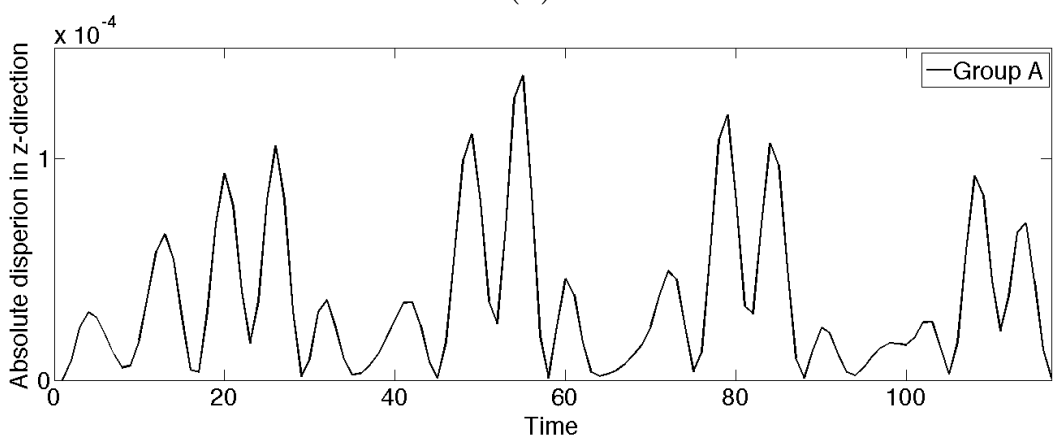

(b)

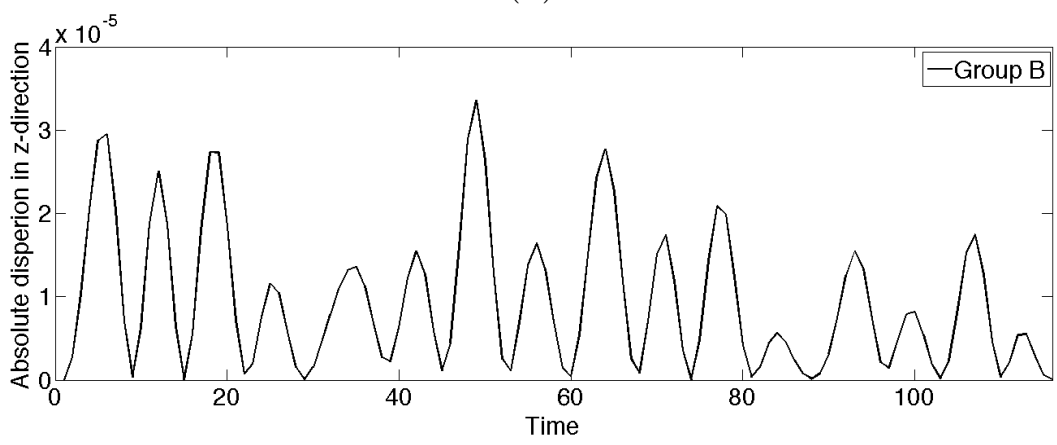

(c)

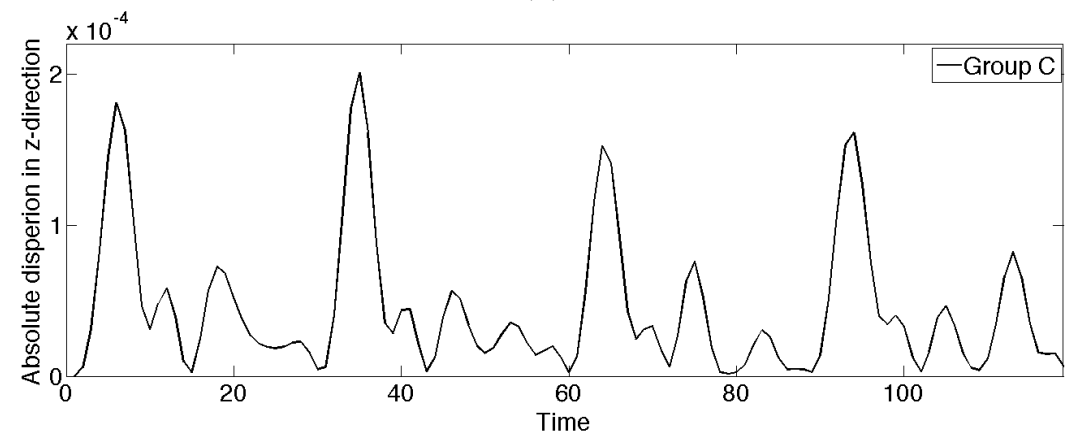

(d)

Figure 19: (a) Initial positions of particle groups A,B,C at the wave field of Exp. T7. (b,c,d) Evolutions of the absolute dispersion of particle groups A,B,C, respectively. 


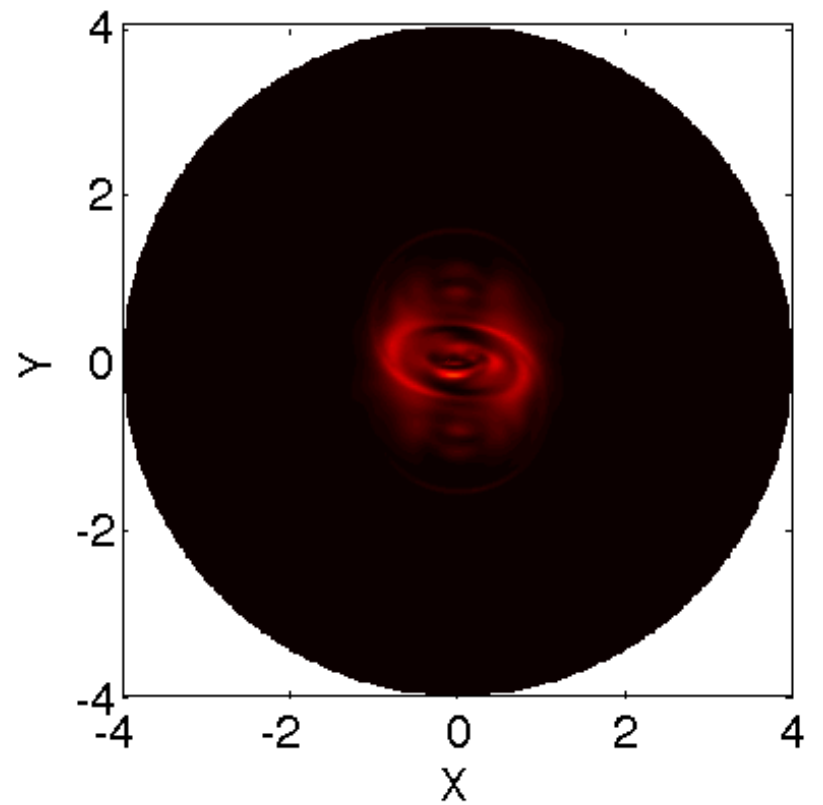

(a)

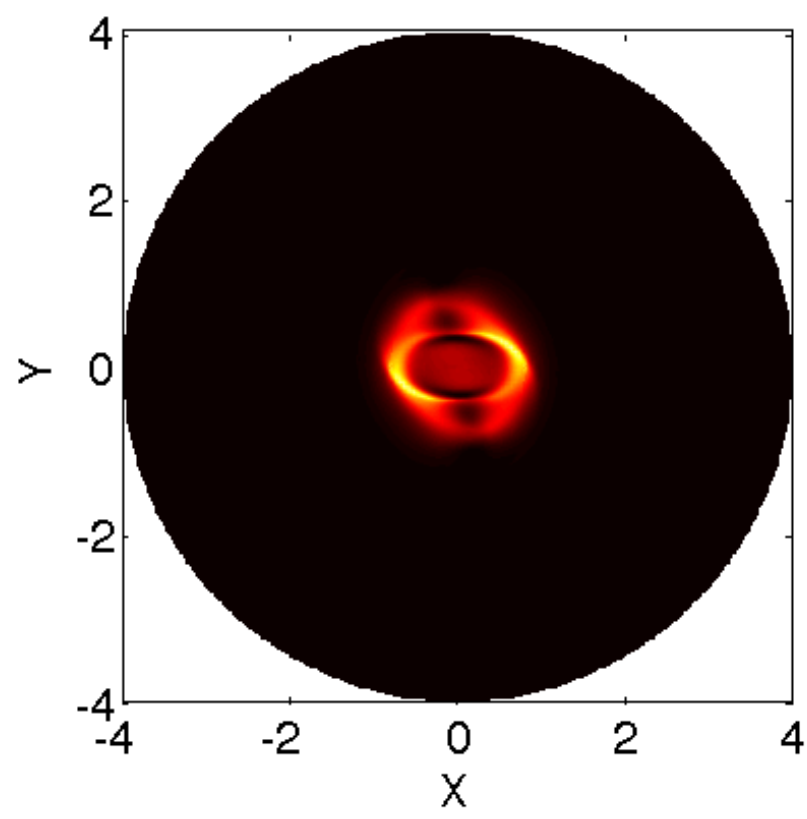

(c)

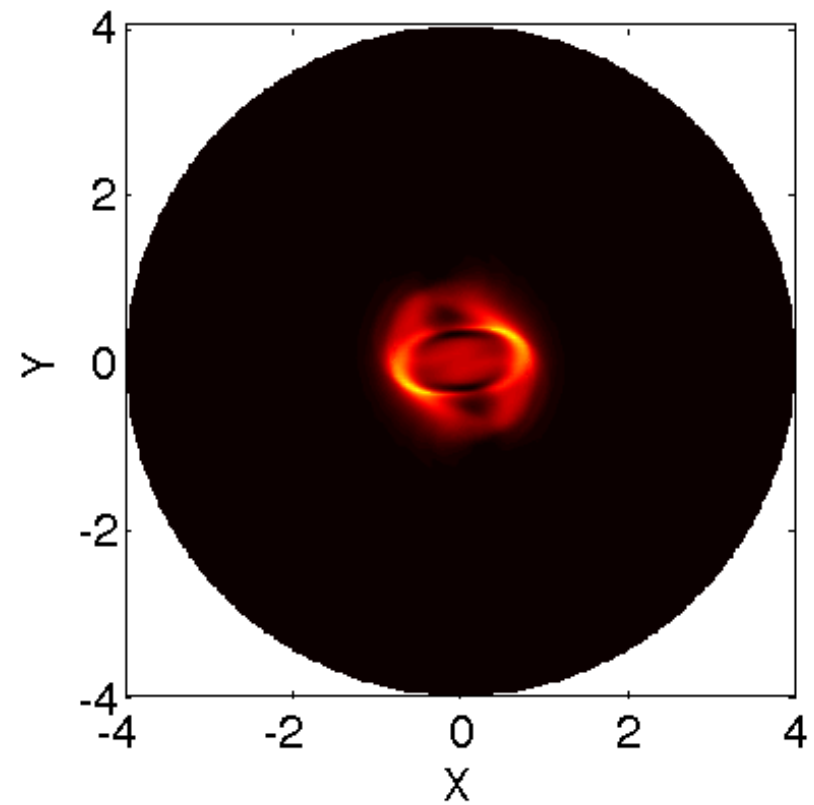

(b)

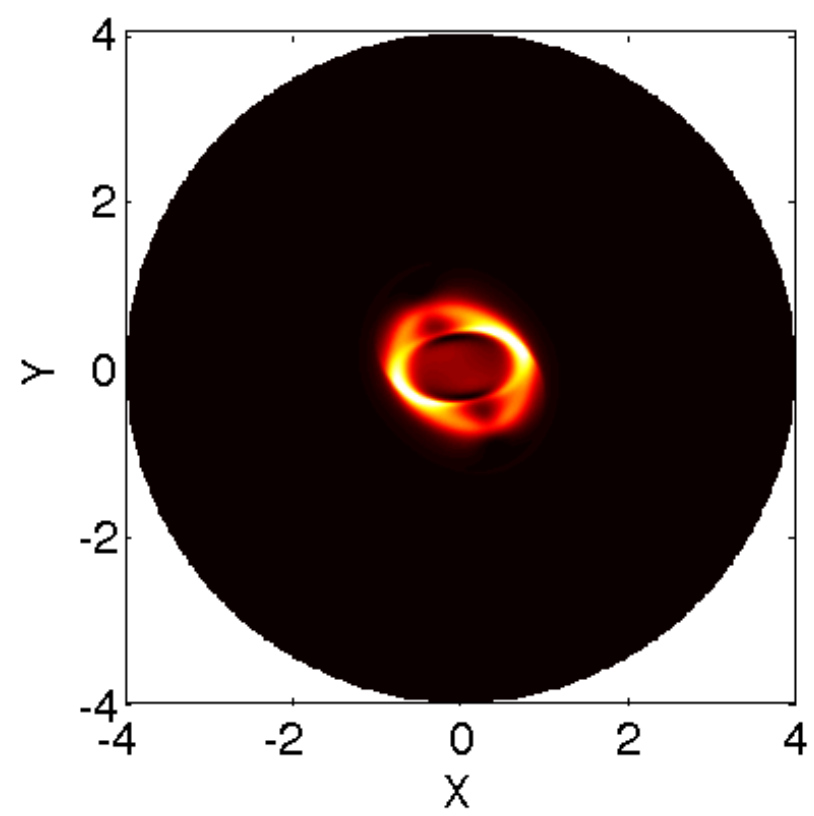

(d)

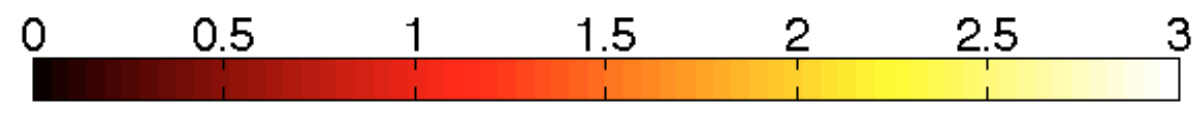

Figure 20: Snapshots of the square of horizontal strain rate on mid-depth horizontal plane in Exps. (a) T7 (b) T4 (c) T3 and (d) T2. 


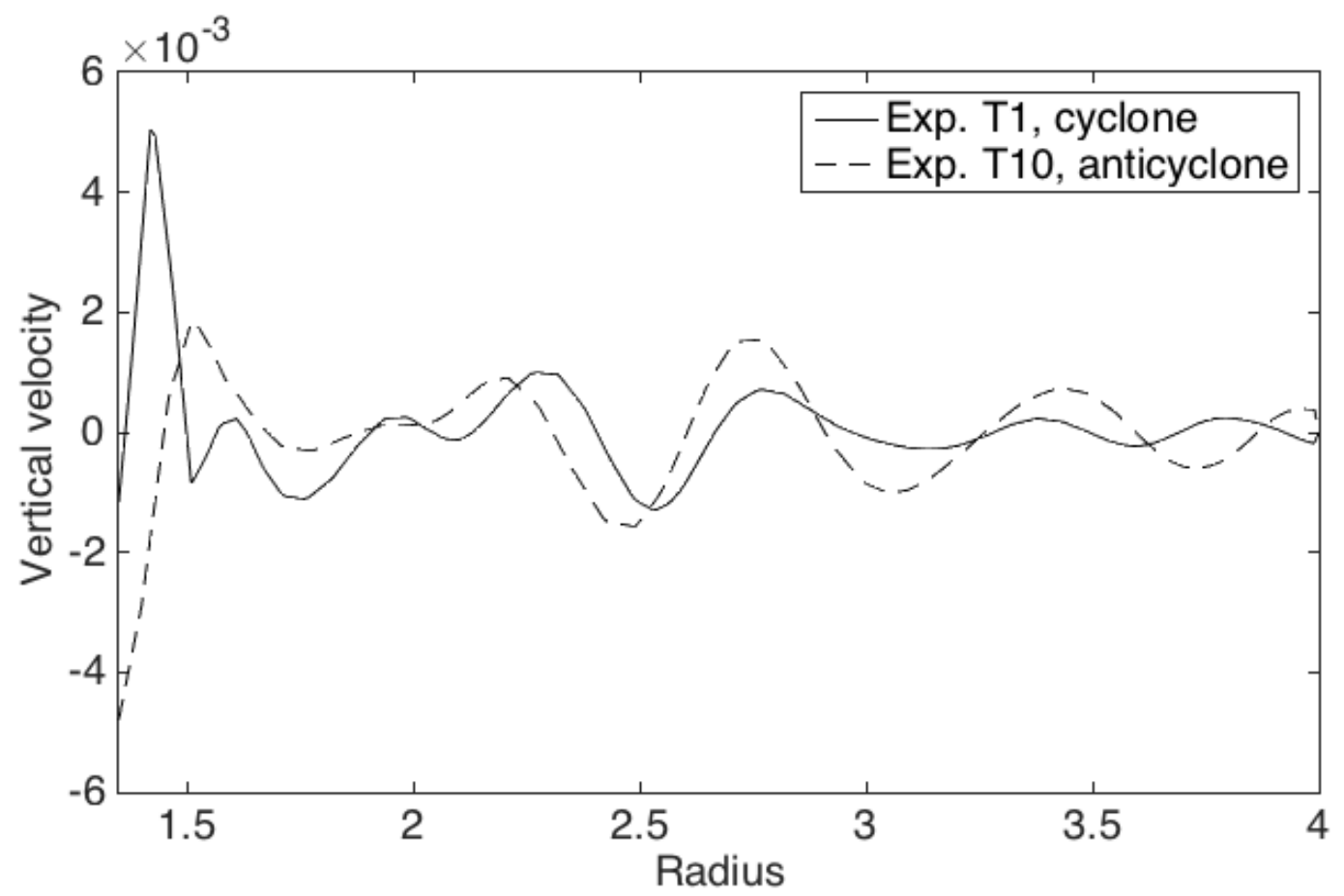

(a)

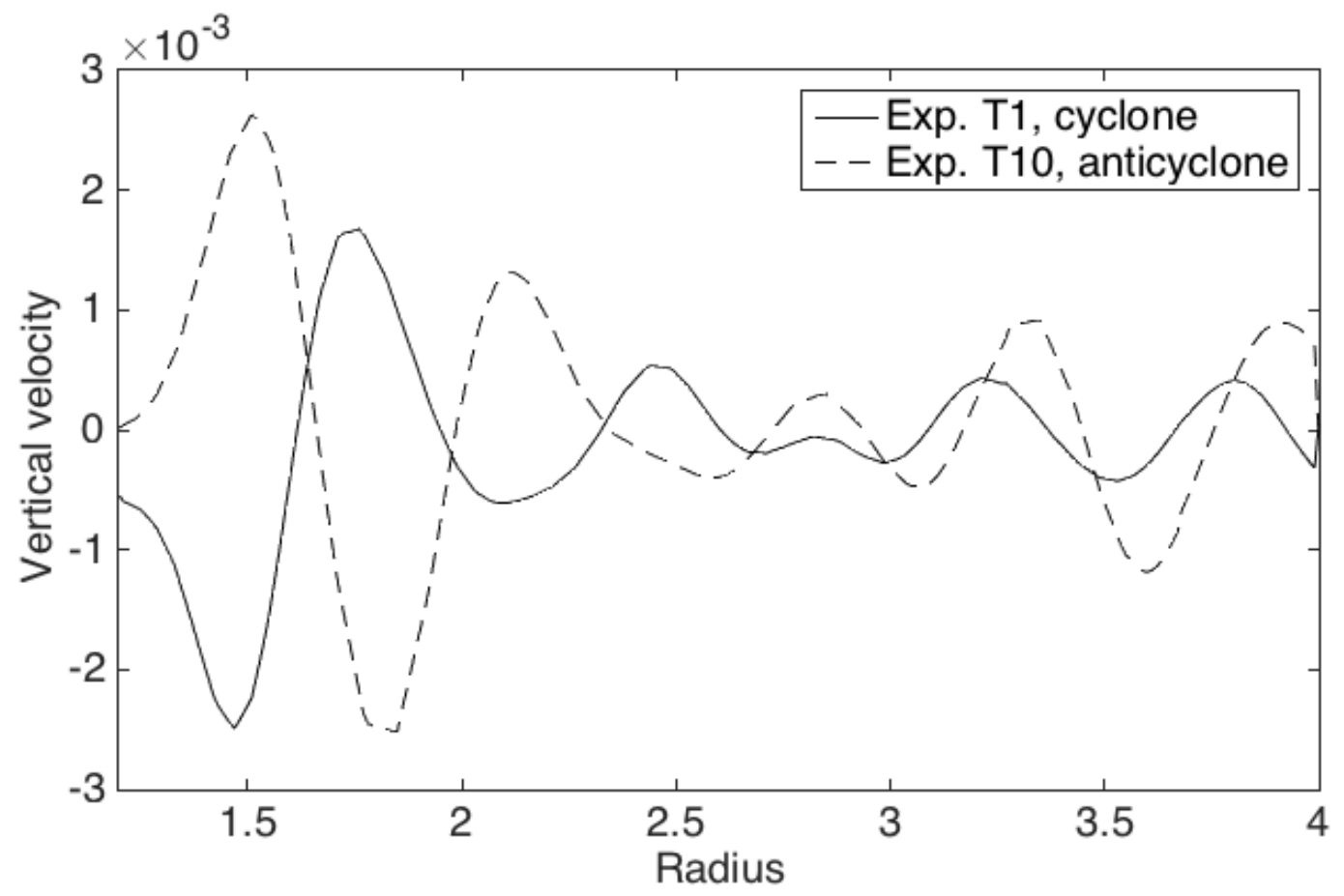

(b)

Figure 21: Vertical velocity in the wave field on the (a) $\theta=0$ and (b) $\theta=\pi / 2 \operatorname{section}(\theta$ is the angle coordinate of the cylindrical frame) at mid-depth in Exps. T1 and T10, at the final stage of vortex evolution. 